\title{
Study of half-sandwich mono and dinuclear complexes of platinum group metals containing pyrazolyl pyridine analogues: Synthesis and spectral characterization
}

\author{
VENKATESWARA RAO ANNA ${ }^{\mathrm{a}}$, KOTA THIRUMALA PRASAD ${ }^{\mathrm{a}}$, PENG WANG $^{\mathrm{b}}$ \\ and KOLLIPARA MOHAN RAO ${ }^{\mathrm{a}, *}$ \\ ${ }^{a}$ Department of Chemistry, North-Eastern Hill University, Shillong 793 022, India \\ ${ }^{b}$ Department of Chemistry, McMaster University, 1280 Main Street West, Hamilton, Ontario L8S 4M1, Canada \\ e-mail:mohanrao59@gmail.com
}

MS received 4 September 2011; revised 25 November 2011; accepted 5 December 2011

\begin{abstract}
The chelating ligands 3-chloro-6-(3-pyridyl-1-pyrazolyl)pyridazine ( $\mathrm{pp}$ - $\mathrm{Cl}$ ) and 3,6-bis(3-pyridyl1-pyrazolyl)pyridazine ( dazine. The mononuclear complexes $\left[\left(\eta^{6} \text {-arene }\right) \mathrm{Ru}(p p-\mathrm{Cl}) \mathrm{Cl}\right]^{+}\left\{\eta^{6}\right.$-arene $=\mathrm{C}_{6} \mathrm{H}_{6}(\mathbf{1}) ; p$ - $\left.{ }^{\mathrm{i}} \mathrm{PrC}_{6} \mathrm{H}_{4} \mathrm{Me}(\mathbf{2})\right\}$, $\left[\left(\eta^{5}-\mathrm{C}_{5} \mathrm{Me}_{5}\right) \mathrm{M}(p p-\mathrm{Cl})\right]^{+}\{\mathrm{M}=\mathrm{Rh}(\mathbf{3}) ; \operatorname{Ir}(\mathbf{4})\}, \quad\left[\left(\eta^{6} \text {-arene }\right) \mathrm{Ru}(b p p p) \mathrm{Cl}\right]^{+}\left\{\eta^{6}\right.$-arene $=\mathrm{C}_{6} \mathrm{H}_{6}$ (5); $p$ $\left.{ }^{i} \mathrm{PrC}_{6} \mathrm{H}_{4} \mathrm{Me}(6)\right\},\left[\left(\eta^{5}-\mathrm{C}_{5} \mathrm{Me}_{5}\right) \mathrm{M}(b p p p)\right]^{+}\{\mathrm{M}=\mathrm{Rh}(\mathbf{7}) ; \mathrm{Ir}(\mathbf{8})\}$ as well as the binuclear complexes $\left[\left\{\left(\eta^{6}-\right.\right.\right.$ arene $\left.) \mathrm{RuCl}\}_{2}(\mathrm{bppp})\right]^{2+}\left\{\eta^{6}\right.$-arene $\left.=\mathrm{C}_{6} \mathrm{H}_{6}(\mathbf{9}) ; p-{ }^{\mathrm{i}} \mathrm{PrC}_{6} \mathrm{H}_{4} \mathrm{Me}(\mathbf{1 0})\right\}$ and $\left[\left\{\left(\eta^{5}-\mathrm{C}_{5} \mathrm{Me}_{5}\right) \mathrm{MCl}\right\}_{2}(b p p p)\right]^{2+}\{\mathrm{M}=$ $\mathrm{Rh}$ (11); Ir (12)\} have been synthesized from 3-chloro-6-(3-pyridyl-1-pyrazolyl)pyridazine ( $\mathrm{pp}$ - $\mathrm{Cl}$ ) or 3,6-bis(3-pyridyl-1-pyrazolyl)pyridazine ( $[\mathrm{Cp} * \mathrm{M}(\mu-\mathrm{Cl}) \mathrm{Cl}]_{2}$, respectively. All complexes were isolated as their hexafluorophosphate salts and characterized by IR, NMR, mass spectrometry and UV-visible spectroscopy. The molecular structures of [2] $\mathrm{PF}_{6}$ and [7] $\mathrm{PF}_{6}$ have been established by single crystal $\mathrm{X}$-ray structure analysis.
\end{abstract}

Keywords. N-donor ligands; mono and binuclear complexes; ruthenium; rhodium; iridium.

\section{Introduction}

Mono and binuclear complexes of platinum group metals containing heterocyclic nitrogen based ligands have received considerable attention owing to their catalytic activities ${ }^{1-7}$ as well as in the development of new biologically active agents. ${ }^{8-12}$ The organometallic complexes of $\eta^{6}$-arene ruthenium, ${ }^{13,14}$ and $\eta^{5}$-Cp* halfsandwich complexes of rhodium and iridium have attracted considerable interest as potential anticancer agents. ${ }^{8-12}$ Another important aspect, especially from the catalytic perspective involves the design of $\mathrm{Ru}=\mathrm{O}$ functional groups and analogues capable of reversibly accepting multiple electrons and protons within the relative potential range. ${ }^{15-17}$ The capacity to modify their environment in order to induce electronic as well as steric effects will allow fabricating tailored catalysis for specific reactions. Inclusion of the six-membered pyridazine ring in the backbone of bppp ligand results in a more pronounced partitioning of the ligand into distinct bidentate domains than in the case with linear polypyridines. This facilitates the formation of mononuclear and binuclear systems. The former has

*For correspondence the potential to behave as metallo-ligands in the development of homo/hetero bimetallic systems. ${ }^{18-21}$ In the present contribution, we have synthesized new nitrogen based ligands such as 3-chloro-6-(3-pyridyl-1pyrazolyl)pyridazine ( $p p-C l$ ) and 3,6-bis(3-pyridyl-1pyrazolyl)pyridazine ( $b p p p$ ) which easily form mono and binuclear complexes with arene ruthenium, $\mathrm{Cp}^{*}$ rhodium and $\mathrm{Cp}^{*}$ iridium complexes $\left(\mathrm{Cp}^{*}=\eta^{5}-\right.$ $\mathrm{C}_{5} \mathrm{Me}_{5}$ ). All these new complexes were characterized by elemental analyses, IR, ${ }^{1} \mathrm{H}-\mathrm{NMR}$, UV-Visible and mass spectrometry as well as $\mathrm{X}$-ray crystallographic analyses for some representative complexes. The ligands which are used in the study are shown in chart 1 .

\section{Experimental}

\subsection{General remarks}

All solvents were dried and distilled prior to use. 3,6-dichloropyridazine (Aldrich), acetylpyridine, pyrazole (Aldrich) were purchased and used as received. $\left[\left(\eta^{6}-\mathrm{C}_{6} \mathrm{H}_{6}\right) \mathrm{Ru}(\mu-\mathrm{Cl}) \mathrm{Cl}\right]_{2}, \quad\left[\left(\eta^{6}-p-{ }^{\mathrm{i}} \mathrm{PrC}_{6} \mathrm{H}_{4} \mathrm{Me}\right) \mathrm{Ru}(\mu-\right.$ $\mathrm{Cl}) \mathrm{Cl}_{2},{ }^{22}$ and $[\mathrm{Cp} * \mathrm{M}(\mu-\mathrm{Cl}) \mathrm{Cl}]_{2}(\mathrm{M}=\mathrm{Rh}, \mathrm{Ir})^{23}$ were 


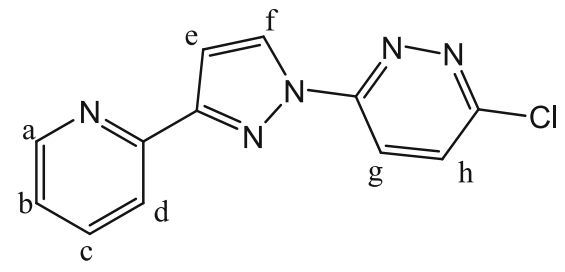

3-Chloro-6-(3-pyridyl-1-pyrazolyl)pyridazine( $p p-\mathrm{Cl})$

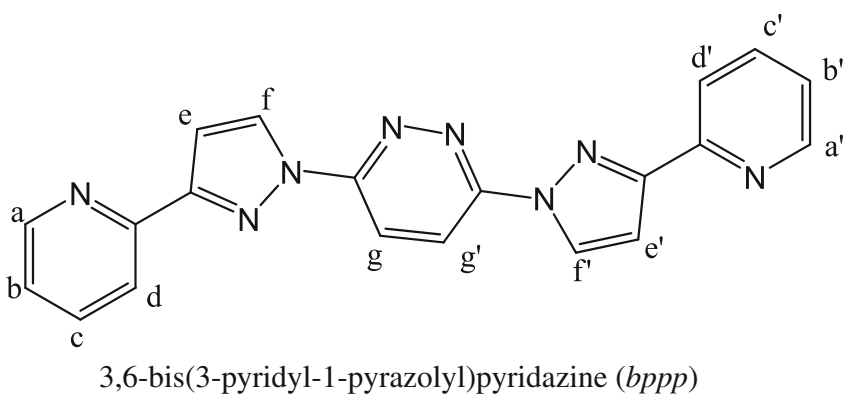

Chart 1. Ligands used in the study.

prepared according to the literature methods. The 3(2-pyridyl)-1H-pyrazole (pypz) has been prepared by using previously described method. ${ }^{24}{ }^{1} \mathrm{H}-\mathrm{NMR}$ spectra were recorded on Bruker-AMX-400 MHz spectrometer. Infrared spectra were recorded as $\mathrm{KBr}$ pellets on a Perkin-Elmer 983 spectrophotometer; elemental analyses of the complexes were performed on a PerkinElmer-2400 CHN/S analyzer. Mass spectra were obtained from Waters ZQ mass spectrometer by ESI method. Absorption spectra were obtained at room temperature using a Perkin-Elmer Lambda UV/Visible spectrophotometer.

\subsection{Preparations of ligands: pp-Cl and bppp}

A mixture of 3,6-dichloropyridazine $(500 \mathrm{mg}, 3.35 \mathrm{mmol})$, 3-(2-pyridyl)-1H-pyrazole (1 g, $6.88 \mathrm{mmol}$ ), potassium carbonate $(1.05 \mathrm{~g}, 7.59 \mathrm{mmol})$ and tetrabutyl ammonium bromide $(1.2 \mathrm{~g})$ in $10 \mathrm{ml}$ of acetone was dissolved. It was stirred and refluxed for $40 \mathrm{~h}$ and cooled to room temperature. Then the reaction mixture was poured in to $100 \mathrm{ml}$ of water, resulted in whitish precipitate; it was filtered off, washed with excess water and air dried. It was purified by chromatography on silica gel using chloroform as the eluent to give the analytically pure ligand 3-chloro-6-(3-pyridyl-1pyrazolyl)pyridazine $(\mathrm{pp}-\mathrm{Cl})(20 \%)$ as a pale yellow colour powder and the second fraction was eluted with chloroform/methanol (10:1) give the analytically pure ligand 3,6-bis(3-pyridyl-1-pyrazolyl)pyridazine ( $(80 \%)$ as a colourless solid.

2.2a 3-chloro-6-(3-pyridyl-1-pyrazolyl)pyridazine (pp$\mathrm{Cl})$ : Pale yellow solid, yield $120 \mathrm{mg}(13 \%){ }^{1} \mathrm{H}$ NMR
(400 MHz, $\left.\mathrm{CDCl}_{3}, \delta\right): 8.77(\mathrm{~d}, 1 \mathrm{H}, J=2.80 \mathrm{~Hz}, \mathrm{Ha})$, $8.68(\mathrm{~d}, 1 \mathrm{H}, J=4.40 \mathrm{~Hz}, \mathrm{Hf}), 8.34(\mathrm{~d}, 1 \mathrm{H}, J=$ $9.20 \mathrm{~Hz}, \mathrm{Hg}$ ), 8.04 (d, 1H, $J=8.00 \mathrm{~Hz}, \mathrm{Hh}), 7.77$ $(\mathrm{dt}, 1 \mathrm{H}, J=1.60 \mathrm{~Hz}, \mathrm{Hd}), 7.63(\mathrm{~d}, 1 \mathrm{H}, J=9.20 \mathrm{~Hz}$, $\mathrm{Hc}), 7.28(\mathrm{dt}, 1 \mathrm{H}, J=1.60 \mathrm{~Hz}, \mathrm{Hb}), 7.18(\mathrm{~d}, 1 \mathrm{H}$, $J=2.40 \mathrm{~Hz}, \mathrm{He})$; ESI-MS (m/z): 258.1 (100\%) [M+1]; UV-Vis $\left\{\right.$ acetonitrile, $\left.\lambda_{\max } \mathrm{nm}\left(\varepsilon 10^{-5} \mathrm{M}^{-1} \mathrm{~cm}^{-1}\right)\right\}: 318$ (0.37).

$2.2 \mathrm{~b}$ 3,6-Bis(3-pyridyl-1-pyrazolyl)pyridazine (bppp): White solid, yield $780 \mathrm{mg}(63 \%){ }^{1} \mathrm{H}$ NMR (400 MHz, $\left.\mathrm{CDCl}_{3}, \delta\right): 8.81\left(\mathrm{~d}, 2 \mathrm{H}, J=2.40 \mathrm{~Hz}, \mathrm{H}_{\mathrm{aa}}{ }^{\prime}\right), 8.71(\mathrm{~d}$, $\left.2 \mathrm{H}, J=4.40 \mathrm{~Hz}, \mathrm{H}_{\mathrm{ff}}{ }^{\prime}\right), 8.52\left(\mathrm{~s}, 2 \mathrm{H}, \mathrm{H}_{\mathrm{gg}}{ }^{\prime}\right), 8.11(\mathrm{~d}$, $\left.2 \mathrm{H}, J=8.00, \mathrm{H}_{\mathrm{dd}}{ }^{\prime}\right), 7.80\left(\mathrm{dt}, 2 \mathrm{H}, J=1.60 \mathrm{~Hz}, \mathrm{H}_{\mathrm{cc}}{ }^{\prime}\right)$, $7.29\left(\mathrm{dt}, 2 \mathrm{H}, J=1.60 \mathrm{~Hz}, \mathrm{H}_{\mathrm{bb}}{ }^{\prime}\right), 7.21(\mathrm{~d}, 2 \mathrm{H}, J=$ $2.80 \mathrm{~Hz}, \mathrm{H}_{\mathrm{ee}}{ }^{\prime}$ ); ESI-MS (m/z): $367.2(100 \%)[\mathrm{M}+1]$; $\mathrm{UV}-$ Vis $\left\{\right.$ acetonitrile, $\left.\lambda_{\max } \mathrm{nm}\left(\varepsilon 10^{-5} \mathrm{M}^{-1} \mathrm{~cm}^{-1}\right)\right\}: 316$ (0.41), $331(0.33)$.

\subsection{General procedure for the preparation}

of the mononuclear complexes $[\mathbf{1}] P F_{6}-[2] P F_{6}$

A mixture of $\left[\left(\eta^{6} \text {-arene }\right) \mathrm{Ru}(\mu-\mathrm{Cl}) \mathrm{Cl}\right]_{2}$ (arene $=$ $\mathrm{C}_{6} \mathrm{H}_{6}$ and $p$ - $\left.{ }^{\mathrm{i}} \mathrm{PrC}_{6} \mathrm{H}_{4} \mathrm{Me}\right)(0.07 \mathrm{mmol})$, ligand $p p-\mathrm{Cl}$ $(0.18 \mathrm{mmol})$ and 2.5 equivalents of $\mathrm{NH}_{4} \mathrm{PF}_{6}$ in dry methanol $(15 \mathrm{ml})$ was stirred at room temperature for $6 \mathrm{~h}$. The precipitate was separated by filtration, washed with cold methanol and diethyl ether to remove excess ligand and dried in vacuo.

2.3a $\quad\left[\left(\eta^{6}-C_{6} H_{6}\right) R u(p p-C l) C l\right] P F_{6} \quad\left([1] P F_{6}\right):$ Orangeyellow solid, yield $75 \mathrm{mg}(87 \%){ }^{1} \mathrm{H}$ NMR $(400 \mathrm{MHz}$, $\left.\mathrm{CD}_{3} \mathrm{CN}, \delta\right): 9.55(\mathrm{~d}, 1 \mathrm{H}, J=5.60 \mathrm{~Hz}), 8.97(\mathrm{~d}, 1 \mathrm{H}$, $J=2.80 \mathrm{~Hz}), 8.72(\mathrm{~d}, 1 \mathrm{H}, J=7.28 \mathrm{~Hz}), 8.45(\mathrm{~m}$, $2 \mathrm{H}), 8.32(\mathrm{t}, 1 \mathrm{H}, J=7.60 \mathrm{~Hz}, 7.60 \mathrm{~Hz}), 7.78(\mathrm{~m}$, 2H), 5.95 (s, 6H); IR (KBr, cm $\left.{ }^{-1}\right)$ : 1604(m), 1408(s),

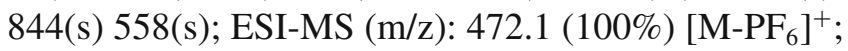
UV-Vis \{acetonitrile, $\left.\lambda_{\max } \mathrm{nm}\left(\varepsilon 10^{-5} \mathrm{M}^{-1} \mathrm{~cm}^{-1}\right)\right\}: 310$ (0.19); Anal. Calc. for $\mathrm{C}_{18} \mathrm{H}_{14} \mathrm{Cl}_{2} \mathrm{~F}_{6} \mathrm{~N}_{5} \mathrm{PRu}$ (617.28): $\mathrm{C}$, 35.02; H, 2.29; N, 11.35. Found: C, 34.72; H, 2.13; N, $11.05 \%$.

$2.3 \mathrm{~b} \quad\left[\left(\eta^{6}-p-{ }^{\mathrm{i}} \mathrm{PrC}_{6} \mathrm{H}_{4} \mathrm{Me}\right) \mathrm{Ru}(\mathrm{pp}-\mathrm{Cl}) \mathrm{Cl}\right] P \mathrm{PF}_{6} \quad\left([\mathbf{2}] P \mathrm{PF}_{6}\right)$ : Dark orange solid, yield $83 \mathrm{mg}(90 \%){ }^{1} \mathrm{H}$ NMR $\left(400 \mathrm{MHz}, \mathrm{CD}_{3} \mathrm{CN}, \delta\right): 9.22(\mathrm{~d}, 1 \mathrm{H}, J=5.60117 \mathrm{~Hz})$, $8.75(\mathrm{~d}, 1 \mathrm{H}, J=4.80 \mathrm{~Hz}), 8.62(\mathrm{~d}, 1 \mathrm{H}, J=3.20 \mathrm{~Hz})$, 8.19-8.12 (m, 3H), $7.66(\mathrm{dd}, 1 \mathrm{H}, J=7.60 \mathrm{~Hz}$, $7.60 \mathrm{~Hz}), 7.40(\mathrm{~d}, 1 \mathrm{H}, J=3.2 \mathrm{~Hz}) 5.72(\mathrm{~d}, 1 \mathrm{H}, J=$ $\left.6.40 \mathrm{~Hz}, \operatorname{Ar}_{p-c y}\right), 5.46\left(\mathrm{~d}, \mathrm{H}, J=6.00 \mathrm{~Hz}, \mathrm{Ar}_{p-c y}\right.$ ), $5.30\left(\mathrm{~d}, 1 \mathrm{H}, J=6.00 \mathrm{~Hz}, \operatorname{Ar}_{p-c y}\right), 5.18(\mathrm{~d}, 1 \mathrm{H}, J=$ $\left.6.00 \mathrm{~Hz}, \mathrm{Ar}_{p-c y}\right), 2.37$ (sept, $\left.1 \mathrm{H}, \mathrm{CH}\left(\mathrm{CH}_{3}\right)_{2}\right), 2.17$ (s, 
$\left.3 \mathrm{H}, \mathrm{Ar}_{p-c y-M e}\right), 1.21$ (d, 3H, $\left.\mathrm{CH}\left(\mathrm{CH}_{3}\right)_{2}\right), 1.18(\mathrm{~d}, 3 \mathrm{H}$, $\left.\mathrm{CH}\left(\mathrm{CH}_{3}\right)_{2}\right)$; IR $\left(\mathrm{KBr}, \mathrm{cm}^{-1}\right): 1629(\mathrm{~m}), 1406(\mathrm{~s}), 844(\mathrm{~s})$, 763(s), 558(s); ESI-MS (m/z): 527.2 (100\%) [M-PF $]^{+}$; UV-Vis \{acetonitrile, $\left.\lambda_{\max } \mathrm{nm}\left(\varepsilon 10^{-5} \mathrm{M}^{-1} \mathrm{~cm}^{-1}\right)\right\}: 309$ (0.28); Anal. Calc. for $\mathrm{C}_{22} \mathrm{H}_{14} \mathrm{Cl}_{2} \mathrm{~F}_{6} \mathrm{~N}_{5} \mathrm{PRu}$ (665.32): $\mathrm{C}$, 39.72; H, 2.12; N, 10.53. Found: C, 39.34; H, 2.01; N, $10.12 \%$.

\subsection{General procedure for the preparation}

of the mononuclear complexes $[3] P F_{6}$ and $[4] P F_{6}$

A mixture of $[\mathrm{Cp} * \mathrm{M}(\mu-\mathrm{Cl}) \mathrm{Cl}]_{2} \quad(\mathrm{M}=\mathrm{Rh}, \quad$ Ir $)$ $(0.07 \mathrm{mmol})$, ligand $\mathrm{pp}-\mathrm{Cl}(0.15 \mathrm{mmol})$ and 2.5 equivalents of $\mathrm{NH}_{4} \mathrm{PF}_{6}$ in dry methanol $(15 \mathrm{ml})$ was refluxed for $4 \mathrm{~h}$. The reaction mixture was cooled over night at room temperature during this time dark yellow colour crystalline compound formed. It was separated by filtration, washed with cold methanol and diethyl ether to remove excess ligand and dried in vacuo.

2.4a $[\mathrm{Cp} * R h(p p-C l) C l] P F_{6}\left([\mathbf{3}] P F_{6}\right):$ Dark yellow in colour, yield $86 \mathrm{mg}(91 \%){ }^{1} \mathrm{H}$ NMR $(400 \mathrm{MHz}$, $\left.\mathrm{CD}_{3} \mathrm{CN}, \delta\right): 8.91(\mathrm{~d}, 1 \mathrm{H}, J=5.20 \mathrm{~Hz}), 8.67(\mathrm{~d}, 1 \mathrm{H}$, $J=4.40 \mathrm{~Hz}), 8.50(\mathrm{~d}, 1 \mathrm{H}, J=7.60 \mathrm{~Hz}), 8.36(\mathrm{~m}$, $3 \mathrm{H}), 7.44(\mathrm{dt}, 1 \mathrm{H}, J=5.20 \mathrm{~Hz}, 6.80 \mathrm{~Hz}), 7.22(\mathrm{~d}$, $1 \mathrm{H}, J=2.4 \mathrm{~Hz}), 2.15\left(\mathrm{~s}, 15 \mathrm{H}, \mathrm{C}_{5} \mathrm{Me}_{5}\right)$; IR $(\mathrm{KBr}$, $\left.\mathrm{cm}^{-1}\right): 1626(\mathrm{~m}), 1458(\mathrm{~s}), 845(\mathrm{~s}), 759(\mathrm{~s}), 558(\mathrm{~s})$; ESI$\mathrm{MS}(\mathrm{m} / \mathrm{z}): 531.3(100 \%)$ [M-PF $\left._{6}\right]^{+}$; UV-Vis \{acetonitrile, $\left.\lambda_{\max } \mathrm{nm}\left(\varepsilon 10^{-5} \mathrm{M}^{-1} \mathrm{~cm}^{-1}\right)\right\}$ : 316 (0.27); Anal. Calc. for $\mathrm{C}_{22} \mathrm{H}_{23} \mathrm{Cl}_{2} \mathrm{~F}_{6} \mathrm{~N}_{5} \mathrm{PRh}$ (676.23): C, 39.07; $\mathrm{H}$, 3.43 ; N, 10.36. Found: C, 38.85; H, 3.24; N, $10.03 \%$.

2.4b $\quad[\mathrm{Cp} * \operatorname{Ir}(p p-C l) C l] P F_{6} \quad\left([4] P F_{6}\right):$ Dark yellow colour, yield $89 \mathrm{mg}(88 \%){ }^{1} \mathrm{H}$ NMR (400 MHz, $\left.\mathrm{CD}_{3} \mathrm{CN}, \delta\right): 9.01(\mathrm{~d}, 1 \mathrm{H}, J=1395.60 \mathrm{~Hz}), 8.71(\mathrm{~d}$, $1 \mathrm{H}, J=4.80 \mathrm{~Hz}), 8.62(\mathrm{~d}, 1 \mathrm{H}, J=6.40 \mathrm{~Hz}), 8.42$ (m, 3H), $7.48(\mathrm{dt}, 1 \mathrm{H}, J=5.32 \mathrm{~Hz}, 5.60 \mathrm{~Hz}), 7.35$ (d, $1 \mathrm{H}, J=4.80 \mathrm{~Hz}), 1.88\left(\mathrm{~s}, 15 \mathrm{H}, \mathrm{C}_{5} \mathrm{Me}_{5}\right)$; IR ( $\mathrm{KBr}$, $\left.\mathrm{cm}^{-1}\right): 1631(\mathrm{~m}), 1495(\mathrm{~s}), 845(\mathrm{~s}), 760(\mathrm{~s}), 558(\mathrm{~s})$; ESI$\mathrm{MS}(\mathrm{m} / \mathrm{z}): 620.9(100 \%)\left[\mathrm{M}^{-P_{6}}\right]^{+}$; UV-Vis \{acetonitrile, $\left.\lambda_{\max } \mathrm{nm}\left(\varepsilon 10^{-5} \mathrm{M}^{-1} \mathrm{~cm}^{-1}\right)\right\}: 318$ (0.29); Anal. Calc. for $\mathrm{C}_{22} \mathrm{H}_{23} \mathrm{Cl}_{2} \mathrm{~F}_{6} \mathrm{IrN}_{5} \mathrm{P}$ (765.54): C, 34.52; H, 3.03; N, 9.15. Found: C, 34.01; H, 2.92; N, $8.95 \%$.

\subsection{General procedure for the preparation} of the mononuclear complexes $[\mathbf{5}] P F_{6}-[\mathbf{6}] P F_{6}$

A mixture of $\left[\left(\eta^{6} \text {-arene }\right) \mathrm{Ru}(\mu-\mathrm{Cl}) \mathrm{Cl}\right]_{2}$ (arene $=\mathrm{C}_{6} \mathrm{H}_{6}$ and $\left.p-{ }^{\mathrm{i}} \mathrm{PrC}_{6} \mathrm{H}_{4} \mathrm{Me}\right) \quad(0.07 \mathrm{mmol})$, ligand $b p p p$ $(0.15 \mathrm{mmol})$ and 2.5 equivalents of $\mathrm{NH}_{4} \mathrm{PF}_{6}$ in dry methanol $(15 \mathrm{ml})$ was stirred at room temperature for
$6 \mathrm{~h}$. The precipitate was separated by filtration, washed with cold methanol and diethyl ether to remove excess ligand and dried in vacuo.

2.5a $\quad\left[\left(\eta^{6}-C_{6} H_{6}\right) R u(b p p p) C l\right] P F_{6} \quad\left([5] P F_{6}\right):$ Brown colour; yield $85 \mathrm{mg}(84 \%){ }^{1} \mathrm{H}$ NMR (400 MHz, $\left.\mathrm{CD}_{3} \mathrm{CN}, \delta\right): 9.48(\mathrm{~d}, 1 \mathrm{H}, J=5.20 \mathrm{~Hz}), 8.68(\mathrm{~d}, 1 \mathrm{H}, J$ $=5.64 \mathrm{~Hz}), 8.62(\mathrm{~d}, 1 \mathrm{H}, J=3.20 \mathrm{~Hz}), 8.32-8.28(\mathrm{~m}$, $5 \mathrm{H}), 7.76(\mathrm{dt}, 2 \mathrm{H}), 7.62(\mathrm{t}, 1 \mathrm{H}, J=3.32 \mathrm{~Hz}), 7.43(\mathrm{~d}, 1$ $\mathrm{H}, J=3.24 \mathrm{~Hz}), 7.26(\mathrm{~d}, 1 \mathrm{H}, J=4.20 \mathrm{~Hz}), 7.20(\mathrm{~d}$, $1 \mathrm{H}, J=4.00 \mathrm{~Hz}), 5.92\left(\mathrm{~s}, 6 \mathrm{H}, \mathrm{C}_{6} \mathrm{H}_{6}\right)$; IR $\left(\mathrm{cm}^{-1}\right): 1614$ (m), 1454 (s), 1437 (s), 844 (s), 788 (s), 558 (s); ESIMS: 580.9 [M+1], 545.2 [M-Cl]; UV-Vis \{acetonitrile, $\left.\lambda_{\max } \mathrm{nm}\left(\varepsilon 10^{-5} \mathrm{M}^{-1} \mathrm{~cm}^{-1}\right)\right\}: 276$ (0.57), 314 (0.92), 417 (0.04); Anal. Calc. for $\mathrm{C}_{26} \mathrm{H}_{20} \mathrm{ClF}_{6} \mathrm{~N}_{8} \mathrm{PRu}$ (725.98): $\mathrm{C}$, 43.01; H, 2.78; N, 15.43. Found: C, 42.65; H, 2.65; N, $15.11 \%$.

$2.5 \mathrm{~b} \quad\left[\left(\eta^{6}-p^{-}{ }^{i} \mathrm{PrC}_{6} \mathrm{H}_{4} \mathrm{Me}\right) \mathrm{Ru}(\mathrm{bppp}) \mathrm{Cl}\right] \mathrm{PF}_{6} \quad\left([\mathbf{6}] P \mathrm{PF}_{6}\right)$ : Orange-yellow solid; yield $89 \mathrm{mg}(83 \%){ }^{1} \mathrm{H}$ NMR (400 MHz, $\left.\mathrm{CD}_{3} \mathrm{CN}, \delta\right): 9.55(\mathrm{~d}, 1 \mathrm{H}, J=5.20 \mathrm{~Hz}), 8.72$ $(\mathrm{d}, 1 \mathrm{H}, J=5.64 \mathrm{~Hz}), 8.68(\mathrm{~d}, 1 \mathrm{H}, J=3.20 \mathrm{~Hz}), 8.20$ $8.18(\mathrm{~m}, 4 \mathrm{H}), 7.68(\mathrm{dt}, 2 \mathrm{H}), 7.42(\mathrm{t}, 1 \mathrm{H}, J=3.32 \mathrm{~Hz})$, $7.38(\mathrm{~d}, 1 \mathrm{H}, J=4.80 \mathrm{~Hz}), 7.22(\mathrm{~d}, 1 \mathrm{H}, J=4.20 \mathrm{~Hz})$, $7.18(\mathrm{~d}, 2 \mathrm{H}, J=5.60 \mathrm{~Hz}), 5.72(\mathrm{~d}, 1 \mathrm{H}, J=6.40 \mathrm{~Hz}$, $\left.\operatorname{Ar}_{\mathrm{p}-\mathrm{cy}}\right), 5.42\left(\mathrm{~d}, 1 \mathrm{H}, J=6.00 \mathrm{~Hz}, \operatorname{Ar}_{\mathrm{p}-\mathrm{cy}}\right), 5.39(\mathrm{~d}$, $\left.1 \mathrm{H}, J=6.00 \mathrm{~Hz}, \mathrm{Ar}_{\mathrm{p}-\mathrm{cy}}\right), 5.29(\mathrm{~d}, 1 \mathrm{H}, J=5.60 \mathrm{~Hz}$, $\mathrm{Ar}_{\mathrm{p}-\mathrm{cy}}$ ), 2.70 (sept, $\left.1 \mathrm{H}, \mathrm{CH}\left(\mathrm{CH}_{3}\right)_{2}\right), 2.33$ (s, 3H, $\left.\mathrm{Ar}_{\mathrm{p}-\mathrm{cy}-\mathrm{Me}}\right), 1.71\left(\mathrm{~d}, 3 \mathrm{H}, 3 \mathrm{~J}=6.20 \mathrm{~Hz}, \mathrm{CH}\left(\mathrm{CH}_{3}\right)_{2}\right)$, $1.69\left(\mathrm{~d}, 3 \mathrm{H}, J=6.80 \mathrm{~Hz}, \mathrm{CH}\left(\mathrm{CH}_{3}\right)_{2}\right)$; $\mathrm{IR}\left(\mathrm{cm}^{-1}\right)$ : 1604(m), 1449(s), 1437(s), 843(s), 783(s), 558(s); ESIMS: $637.1[\mathrm{M}+1], 602.1$ [M-Cl]; UV-Vis \{acetonitrile $\left.\lambda_{\max } \mathrm{nm}\left(\varepsilon 10^{-5} \mathrm{M}^{-1} \mathrm{~cm}^{-1}\right)\right\}: 274(0.57), 313$ (0.89), 418 (0.05); Anal. Calc. for $\mathrm{C}_{30} \mathrm{H}_{28} \mathrm{ClF}_{6} \mathrm{~N}_{8} \mathrm{PRu}$ (782.08): C, 46.07; H, 3.61; N, 14.33. Found: C, 45.64; H, 3.53; $\mathrm{N}, 14.11 \%$.

\subsection{General procedure for the preparation}

of the mononuclear complexes $[7] P F_{6}$ and $[\mathbf{8}] P F_{6}$

A mixture of $\left[\mathrm{Cp}^{*} \mathrm{M}(\mu-\mathrm{Cl}) \mathrm{Cl}\right]_{2} \quad(\mathrm{M}=\mathrm{Rh}, \quad \mathrm{Ir})$ $(0.07 \mathrm{mmol})$, ligand $\operatorname{sppp}(0.15 \mathrm{mmol})$ and 2.5 equivalents of $\mathrm{NH}_{4} \mathrm{PF}_{6}$ in dry methanol $(15 \mathrm{ml})$ was refluxed for $4 \mathrm{~h}$. The reaction mixture was cooled over night at room temperature during this time dark yellow colour crystalline compound formed. It was separated by filtration, washed with cold methanol and diethyl ether to remove excess ligand and dried under vacuum.

2.6a $\left[\mathrm{Cp} * R h(\right.$ bppp $) \mathrm{Cl} \mathrm{PF} \mathrm{F}_{6} \quad\left([7] P F_{6}\right):$ Dark yellow colour, yield $96 \mathrm{mg}(84 \%){ }^{1} \mathrm{H}$ NMR (400 MHz, $\left.\mathrm{CD}_{3} \mathrm{CN}, \delta\right): 9.39(\mathrm{~d}, 1 \mathrm{H}, J=5.20 \mathrm{~Hz}), 8.68(\mathrm{~d}, 1 \mathrm{H}$, $J=5.40 \mathrm{~Hz}), 8.54(\mathrm{~d}, 1 \mathrm{H}, J=3.64 \mathrm{~Hz}), 8.18-8.10$ 
(m, 4H), $7.61(\mathrm{dt}, 2 \mathrm{H}), 7.48(\mathrm{~d}, 1 \mathrm{H}, J=3.60 \mathrm{~Hz}), 7.32$ $(\mathrm{d}, 1 \mathrm{H}, J=4.8 \mathrm{~Hz}), 7.22(\mathrm{~d}, 1 \mathrm{H}, J=4.20 \mathrm{~Hz}), 7.16$ (d, $2 \mathrm{H}, J=3.64 \mathrm{~Hz}), 2.11\left(\mathrm{~s}, 15 \mathrm{H}, \mathrm{C}_{5} \mathrm{Me}_{5}\right.$ ); ESIMS (m/z): 639.2 (100\%) [M-PF $]^{+}$; IR $\left(\mathrm{KBr}, \mathrm{cm}^{-1}\right)$ : 1626(m), 1458(s), 845(s), 759(s), 558(s); UV-Vis \{acetonitrile, $\left.\lambda_{\max } \mathrm{nm}\left(\varepsilon 10^{-5} \mathrm{M}^{-1} \mathrm{~cm}^{-1}\right)\right\}: 276(0.57), 315$ (0.87), 419 (0.04); Anal. Calc. for $\mathrm{C}_{30} \mathrm{H}_{29} \mathrm{ClF}_{6} \mathrm{~N}_{8} \mathrm{PRh}$ (784.93): C, 45.91; H, 3.72; N, 14.28. Found: C, 45.54; $\mathrm{H}, 3.63 ; \mathrm{N}, 14.01 \%$.

$2.6 \mathrm{~b} \quad\left[\left(\eta^{5}-C_{5} M e_{5}\right) \operatorname{Ir}(\right.$ bppp $\left.) C l\right] P F_{6} \quad\left([\mathbf{8}] P F_{6}\right):$ Dark yellow in colour, yield $95 \mathrm{mg}(83 \%){ }^{1} \mathrm{H}$ NMR (400 MHz, $\left.\mathrm{CD}_{3} \mathrm{CN}, \delta\right): 9.40(\mathrm{~d}, 1 \mathrm{H}, J=5.32 \mathrm{~Hz}$ ), $8.72(\mathrm{~d}, 1 \mathrm{H}, J=5.40 \mathrm{~Hz}), 8.62(\mathrm{~d}, 1 \mathrm{H}, J=3.64 \mathrm{~Hz}$ ), 8.20-8.14 (m, 4H), $7.65(\mathrm{dt}, 2 \mathrm{H}), 7.48(\mathrm{~d}, 1 \mathrm{H}, J=$ $3.60 \mathrm{~Hz}), 7.31(\mathrm{~d}, 1 \mathrm{H}, J=4.8 \mathrm{~Hz}), 7.18(\mathrm{~d}, 1 \mathrm{H}, J$ $=4.80 \mathrm{~Hz}), 7.15(\mathrm{~d}, 2 \mathrm{H}, J=3.60 \mathrm{~Hz}), 1.98(\mathrm{~s}, 15 \mathrm{H}$,

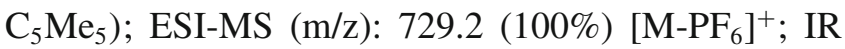
$\left(\mathrm{KBr}, \mathrm{cm}^{-1}\right)$ : 1631(m), 1495(s), 845(s) 760(s), 558(s); UV-Vis \{acetonitrile, $\left.\lambda_{\max } \mathrm{nm}\left(\varepsilon 10^{-5} \mathrm{M}^{-1} \mathrm{~cm}^{-1}\right)\right\}$ : 277 (0.58), 314 (0.95), 423 (0.04); Anal. Calc. for $\mathrm{C}_{30} \mathrm{H}_{29} \mathrm{ClF}_{6} \mathrm{IrN}_{8} \mathrm{P}$ (874.24): C, 41.22; H, 3.34; N, 12.82. Found: C, 40.95; H, 3.24; N, $12.65 \%$.

\subsection{General procedure for the syntheses}

of the dinuclear complexes $[\mathbf{9}]\left(P F_{6}\right)_{2}$ to $[\mathbf{1 0}]\left(P F_{6}\right)_{2}$

A mixture of $\left[\left(\eta^{6} \text {-arene }\right) \mathrm{Ru}(\mu-\mathrm{Cl}) \mathrm{Cl}\right]_{2}$ (arene $=\mathrm{C}_{6} \mathrm{H}_{6}$ and $p$ - $\left.{ }^{\mathrm{i}} \mathrm{PrC}_{6} \mathrm{H}_{4} \mathrm{Me}\right)(0.10 \mathrm{mmol})$ and $\operatorname{loppp}(0.10 \mathrm{mmol})$ was suspended in methanol $(20 \mathrm{ml})$ and stirred at room temperature for $6 \mathrm{~h}$. Then, $\mathrm{NH}_{4} \mathrm{PF}_{6}(46 \mathrm{mg}, 0.25 \mathrm{mmol})$ was added to the reaction mixture and further stirred for $3 \mathrm{~h}$. The precipitate was filtered, washed with methanol and diethylether $(3 \times 10 \mathrm{ml})$ and dried in vacuo.

2.7a $\quad\left[\left\{\left(\eta^{6}-C_{6} H_{6}\right) R u C l\right\}_{2}(\mu-b p p p)\right]\left(P F_{6}\right)_{2} \quad\left([9]\left(P F_{6}\right)_{2}\right)$ : Orange-yellow solid; yield $91 \mathrm{mg}(85 \%){ }^{1} \mathrm{H}$ NMR $\left(400 \mathrm{MHz}, \mathrm{CD}_{3} \mathrm{CN}, \delta\right): 9.34(\mathrm{~d}, 2 \mathrm{H}, J=4.20 \mathrm{~Hz}$ ), $9.14(\mathrm{~s}, 2 \mathrm{H}), 8.81(\mathrm{~d}, 2 \mathrm{H}, J=2.40 \mathrm{~Hz}), 8.24(\mathrm{~d}, 2 \mathrm{H}$, $J=6.80 \mathrm{~Hz}), 7.72(\mathrm{t}, 2 \mathrm{H}, J=5.60,4.80 \mathrm{~Hz}), 7.50(\mathrm{~d}$, $2 \mathrm{H}, J=2.40 \mathrm{~Hz}), 7.36(\mathrm{~d}, 2 \mathrm{H}, J=4.80 \mathrm{~Hz}), 5.75(\mathrm{~s}$, $\left.12 \mathrm{H}, \mathrm{C}_{6} \mathrm{H}_{6}\right)$; IR $\left(\mathrm{cm}^{-1}\right)$ : $1614(\mathrm{~m}), 1454$ (s), 1437 (s), 844 (s), 788 (s), 558 (s); ESI-MS: 940.6[ $\left.\mathrm{M}^{2+}+\mathrm{PF}_{6}^{-}\right]^{+}$; UV-Vis \{acetonitrile, $\left.\lambda_{\max } \mathrm{nm}\left(\varepsilon 10^{-5} \mathrm{M}^{-1} \mathrm{~cm}^{-1}\right)\right\}$ : 278 (0.54), 312 (0.83), 408 (0.04); Anal. Calc. for $\mathrm{C}_{32} \mathrm{H}_{26} \mathrm{Cl}_{2} \mathrm{~F}_{12} \mathrm{~N}_{8} \mathrm{P}_{2} \mathrm{Ru}_{2}$ (1085.58): $\mathrm{C}, 35.40 ; \mathrm{H}, 2.41 ; \mathrm{N}$, 10.32. Found: C, 35.06; H, 2.25; N, $10.02 \%$.

$2.7 \mathrm{~b} \quad\left[\left\{\left(\eta^{6}-p^{-}{ }^{i} \mathrm{PrC}_{6} \mathrm{H}_{4} \mathrm{Me}\right) \mathrm{RuCl}\right\}_{2}(\mu-b p p p)\right]\left(\mathrm{PF}_{6}\right)_{2}([\mathbf{1 0}]$ $\left.\left(\mathrm{PF}_{6}\right)_{2}\right)$ : Orange-yellow solid; yield $99 \mathrm{mg}(84 \%){ }^{1} \mathrm{H}$
NMR (400 MHz, $\left.\mathrm{CD}_{3} \mathrm{CN}, \delta\right): 9.55(\mathrm{~d}, 2 \mathrm{H}, J=$ $5.60 \mathrm{~Hz}), 9.37(\mathrm{~s}, 2 \mathrm{H}), 9.14(\mathrm{~d}, 2 \mathrm{H}, J=2.80 \mathrm{~Hz}), 8.52$ $(\mathrm{d}, 2 \mathrm{H}, J=7.60 \mathrm{~Hz}), 8.48(\mathrm{t}, 2 \mathrm{H}, J=7.20,7.60 \mathrm{~Hz})$, $8.36(\mathrm{t}, 2 \mathrm{H}), 7.86(\mathrm{~d}, 2 \mathrm{H}, J=2.40 \mathrm{~Hz}), 6.10(\mathrm{~d}, 2 \mathrm{H}, J$ $\left.=6.00 \mathrm{~Hz}, \mathrm{Ar}_{\mathrm{p}-\mathrm{cy}}\right), 5.81\left(\mathrm{~d}, 2 \mathrm{H}, J=6.00 \mathrm{~Hz}, \mathrm{Ar}_{\mathrm{p}-\mathrm{cy}}\right)$, $5.69\left(\mathrm{~d}, 2 \mathrm{H}, J=6.00 \mathrm{~Hz}, \mathrm{Ar}_{\mathrm{p}-\mathrm{cy}}\right), 5.54(\mathrm{~d}, 2 \mathrm{H}, J=$ $\left.6.00 \mathrm{~Hz}, \mathrm{Ar}_{\mathrm{p}-\mathrm{cy}}\right), 2.68$ (sept, $\left.2 \mathrm{H}, \mathrm{CH}\left(\mathrm{CH}_{3}\right)_{2}\right), 2.25$ (s, $\left.6 \mathrm{H}, \mathrm{Ar}_{\mathrm{p}-\mathrm{cy}-\mathrm{Me}}\right), 1.18\left(\mathrm{~d}, 3 \mathrm{H}, J=6.20 \mathrm{~Hz}, \mathrm{CH}\left(\mathrm{CH}_{3}\right)_{2}\right)$, 1.12 (d, $\left.3 \mathrm{H}, J=6.80 \mathrm{~Hz}, \mathrm{CH}\left(\mathrm{CH}_{3}\right)_{2}\right)$; IR $\left(\mathrm{cm}^{-1}\right)$ : 1604(m), 1449(s), 1437(s), 843(s), 783(s), 558(s); ESI-MS: $1052.6\left[\mathrm{M}^{2+}+\mathrm{PF}_{6}^{-}\right]^{+}$; UV-Vis \{acetonitrile, $\left.\lambda_{\max } \mathrm{nm}\left(\varepsilon 10^{-5} \mathrm{M}^{-1} \mathrm{~cm}^{-1}\right)\right\}: 276(0.57), 314(0.91)$, 410 (0.04); Anal. Calc. for $\mathrm{C}_{40} \mathrm{H}_{42} \mathrm{Cl}_{2} \mathrm{~F}_{12} \mathrm{~N}_{8} \mathrm{P}_{2} \mathrm{Ru}_{2}$ (1197.79): C, 40.11; H, 3.53; N, 9.36. Found: C, 39.81; $\mathrm{H}, 3.42 ; \mathrm{N}, 9.12 \%$.

\subsection{General procedure for the syntheses}

of the dinuclear complexes $[\mathbf{1 1}]\left(P F_{6}\right)_{2}$ to $[\mathbf{1 2}]\left(P F_{6}\right)_{2}$

A mixture of $[\mathrm{Cp} * \mathrm{M}(\mu-\mathrm{Cl}) \mathrm{Cl}]_{2}(\mathrm{M}=\mathrm{Rh}$ and $\mathrm{Ir})$ $(0.10 \mathrm{mmol})$ and bppp $(0.10 \mathrm{mmol})$ was suspended in methanol $(20 \mathrm{ml})$ and refluxed for $5 \mathrm{~h}$. Then, $\mathrm{NH}_{4} \mathrm{PF}_{6}$ $(23 \mathrm{mg}, 0.13 \mathrm{mmol}$ ) was added to the reaction mixture and further refluxed for an hour. The precipitate observed was filtered, washed with methanol and diethylether $(3 \times 10 \mathrm{ml})$ and dried in vacuo.

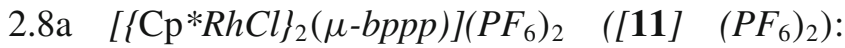
Dark yellow colour, yield $101 \mathrm{mg}(84 \%){ }^{1} \mathrm{H}$ NMR (400 MHz, $\mathrm{CD}_{3} \mathrm{CN}, \delta$ ): 9.38 (d, $2 \mathrm{H}, J=5.40 \mathrm{~Hz}$ ), $9.17(\mathrm{~s}, 2 \mathrm{H}), 8.81(\mathrm{~d}, 2 \mathrm{H}, J=2.32 \mathrm{~Hz}), 8.24$ $(\mathrm{d}, 2 \mathrm{H}, J=6.80 \mathrm{~Hz}), 7.75(\mathrm{t}, 2 \mathrm{H}, J=5.20$, $4.80 \mathrm{~Hz}), 7.52(\mathrm{~d}, 2 \mathrm{H}, J=2.32 \mathrm{~Hz}), 7.37$ (d, $2 \mathrm{H}, J=$ $4.32 \mathrm{~Hz}), 2.05$ (s, 30H, $\left.\mathrm{C}_{5} \mathrm{Me}_{5}\right)$; IR $\left(\mathrm{cm}^{-1}\right)$ : 1604(m), 1449(s), 1437(s), 843(s), 783(s), 558(s); ESI-MS: $1058.6\left[\mathrm{M}^{2+}+\mathrm{PF}_{6}^{-}\right]^{+}$; UV-Vis \{acetonitrile, $\lambda_{\max } \mathrm{nm}$ $\left.\left(\varepsilon 10^{-5} \mathrm{M}^{-1} \mathrm{~cm}^{-1}\right)\right\}: 276$ (0.39), 314 (0.67), 420 (0.04); Anal. Calc. for $\mathrm{C}_{40} \mathrm{H}_{44} \mathrm{Cl}_{2} \mathrm{~F}_{12} \mathrm{~N}_{8} \mathrm{P}_{2} \mathrm{Rh}_{2}$ (1203.48): C, 39.92; H, 3.69; N, 9.31. Found: C, 39.65; H, 3.51; N, $9.01 \%$.

$2.8 \mathrm{~b} \quad\left[\left\{\mathrm{Cp} * \operatorname{IrCl}_{2}(\mu-b p p p)\right]\left(\mathrm{PF}_{6}\right)_{2} \quad\left([\mathbf{1 2}] \quad\left(P F_{6}\right)_{2}\right)\right.$ : Dark yellow colour, yield $105 \mathrm{mg}(86 \%){ }^{1} \mathrm{H}$ NMR $\left(400 \mathrm{MHz}, \mathrm{CD}_{3} \mathrm{CN}, \delta\right): 9.31(\mathrm{~d}, 2 \mathrm{H}, J=4.36 \mathrm{~Hz})$, $9.14(\mathrm{~s}, 2 \mathrm{H}), 8.78(\mathrm{~d}, 2 \mathrm{H}, J=2.80 \mathrm{~Hz}), 8.21(\mathrm{~d}, 2 \mathrm{H}$, $J=6.32 \mathrm{~Hz}), 7.72(\mathrm{t}, 2 \mathrm{H}, J=5.60,4.80 \mathrm{~Hz}), 7.50(\mathrm{~d}$, $2 \mathrm{H}, J=2.40 \mathrm{~Hz}), 7.36(\mathrm{~d}, 2 \mathrm{H}, J=4.80 \mathrm{~Hz}), 1.99(\mathrm{~s}$, $\left.15 \mathrm{H}, \mathrm{C}_{5} \mathrm{Me}_{5}\right)$; IR $\left(\mathrm{cm}^{-1}\right)$ : 1604(m), 1449(s), 1437(s), 843(s), 783(s), 558(s); ESI-MS: $1236.2\left[\mathrm{M}^{2+}+\mathrm{PF}_{6}^{-}\right]^{+}$; UV-Vis \{acetonitrile, $\left.\lambda_{\max } \mathrm{nm}\left(\varepsilon 10^{-5} \mathrm{M}^{-1} \mathrm{~cm}^{-1}\right)\right\}$ : 273 (0.46), 313 (0.71), 418 (0.04); Anal. Calc. for 
$\mathrm{C}_{40} \mathrm{H}_{44} \mathrm{Cl}_{2} \quad \mathrm{~F}_{12} \mathrm{Ir}_{2} \mathrm{~N}_{8} \mathrm{P}_{2}$ (1382.1): C, 34.76; H, 3.21; N, 8.11. Found: C, 34.61; H, 3.25; N, 8.01\%.

\subsection{Single crystal X-ray structure analyses}

X-ray quality crystals of complexes 2 and 7 were grown by slow diffusion of hexane in dichloromethane/ acetonitrile solution of corresponding complexes. The X-ray intensity data were measured at 293(2) K on a Bruker Apex II CCD area detector employing graphite monochromated using Mo- $K \alpha$ radiation $(\lambda=0.71073 \AA$ ). An empirical absorption correction was made by modelling a transmission surface by spherical harmonics employing equivalent reflections with $\mathrm{I}>2 \sigma$ (I) using the program SADBAS. ${ }^{25}$ The structures were solved by direct methods using the program SHELXS 97 and refined by full matrix least squares base on $F^{2}$ using the program SHELXL-97. ${ }^{26}$ The weighting scheme used was $W=1 /\left[\sigma 2\left(F_{02}\right)+0.0311 P_{2}+3.5016 P\right]$ where $P=\left(F_{02}+2 F_{c 2}\right) / 3$. Non-hydrogen atoms were refined anisotropically and hydrogen atoms were refined using a 'riding' model. Refinement converged at a final $R=$ 0.0423 and 0.0454 (for complexes 2 and 7, respectively, for observed data $F^{2}$ ), and $w R 2=0.0804$ and 0.0667 (for complexes 2 and 7, respectively, for unique data $F^{2}$ ). Regarding complex 7 molecular structure, we have encountered two difficulties, they are; (i) one is assign- ing $\mathrm{N}(8)$ and $\mathrm{C}(17)$, and another one is (ii) occupancy of the solvent molecule acetonitrile. The matter regarding an ambiguity in assigning $\mathrm{N}(8)$ and $\mathrm{C}(17)$, exchanging the atoms did not improve the refinement. So the labelling was done in analogy with other compounds and represents the most probable orientation of the molecular fragment. The second factor is now explained on the following lines: the central atom of solvent molecule with occupancy of $\mathrm{C}$ is 0.5 . Since the solvent molecule is acetonitrile, the two heavy peripheral atoms are $\mathrm{N}$ and $\mathrm{C}$. It appears that we cannot differentiate between $\mathrm{N}$ and $\mathrm{C}$, which can be due to the random orientation of $\mathrm{N}$ and $\mathrm{C}$ (they exchange the sites). We also could not locate the $\mathrm{H}$ atoms associated with the peripheral $\mathrm{C}$ atom. This peripheral site was treated as a 50/50 mixture of $\mathrm{C}$ and $\mathrm{N}$, which consider the occupancy of this site yields one molecule of acetonitrile (per two molecules of the complex). The distance between the central $\mathrm{C}$ atom and peripheral atoms is $1.55 \AA$. While other solvent molecules may be present, only acetonitrile molecule has been located from the residual density map. Its presence was manifested by a large residual density, and its introduction lowered the $R$ value from 5.55 to $4.43 \%$. Details of crystallographic data collection parameters and refinement are summarized in table 1. Selected bond lengths and angles are given in table 2 .

Table 1. Crystallographic and structure refinement parameters for compounds $[\mathbf{1}] \mathrm{PF}_{6}$ and $[7] \mathrm{PF}_{6}$.

\begin{tabular}{|c|c|c|}
\hline Complex & {$[2] \mathrm{PF}_{6}$} & {$[7] \mathrm{PF}_{6}$} \\
\hline Chemical formula & $\mathrm{C}_{22} \mathrm{H}_{22} \mathrm{Cl}_{2} \mathrm{~F}_{6} \mathrm{~N}_{5} \mathrm{PRu}$ & $\mathrm{C}_{30} \mathrm{H}_{29} \mathrm{ClF}_{6} \mathrm{~N}_{8}$ PRh.0.5 $\mathrm{CH}_{3} \mathrm{CN}$ \\
\hline Crystal system & Monoclinic & Monoclinic \\
\hline Formulae weight & 673.39 & 803.96 \\
\hline Wavelength $(\AA \stackrel{\circ}{)})$ & 0.71073 & 0.71073 \\
\hline Space group & $\mathrm{C}-2 / \mathrm{c}$ & $\mathrm{P} 21 / n$ \\
\hline Crystal colour and shape & Plate, yellow & Plate, yellow \\
\hline Crystal size $(\mathrm{mm})$ & $0.28 \times 0.15 \times 0.09$ & $0.22 \times 0.14 \times 0.12$ \\
\hline$a(\AA)$ & $10.1768(8)$ & $8.5156(17)$ \\
\hline$b(\AA)$ & $22.7250(16)$ & $19.095(4)$ \\
\hline$c(\AA)$ & $22.133(2)$ & $22.334(5)$ \\
\hline$\beta\left(^{\circ}\right)$ & $97.646(7)$ & $90.46(3)$ \\
\hline$V\left(\AA^{3}\right)$ & $5073.2(7)$ & $3631.5(13)$ \\
\hline$Z$ & 8 & 4 \\
\hline$T(K)$ & $293(2)$ & $293(2)$ \\
\hline$D_{x}\left(\mathrm{~g} / \mathrm{cm}^{3}\right)$ & 1.763 & 1.470 \\
\hline$\mu\left(\mathrm{mm}^{-1}\right)$ & 0.959 & 0.65 \\
\hline Scan range $\left(^{\circ}\right)$ & $1.79<\theta<24.65$ & $2.11<\theta<20.00$ \\
\hline Unique reflections & 4000 & 3331 \\
\hline Reflections used $[\mathrm{I}>2 \sigma(\mathrm{I})]$ & 2291 & 1042 \\
\hline$R_{\text {int }}$ & 0.0646 & 0.189 \\
\hline Final $\mathrm{R}$ indices $[\mathrm{I}>2 \sigma(\mathrm{I})]$ & $0.0423, w R 20.0803$ & $0.0443, w R_{2} 0.0626$ \\
\hline$R$ indices (all data) & $0.0862, w R 20.0863$ & $0.1881, w R_{2} 0.0894$ \\
\hline Goodness-of-fit & 0.824 & 0.534 \\
\hline $\operatorname{Max}, \operatorname{Min}\left(\mathrm{e} \AA^{-3}\right)$ & $0.696,-0.718$ & $0.284,-0.261$ \\
\hline
\end{tabular}


Table 2. Selected bond lengths $(\AA)$ and angles $\left(^{\circ}\right)$ for compounds $[1] \mathrm{PF}_{6}$ and $[7] \mathrm{PF}_{6}$.

\begin{tabular}{|c|c|c|c|}
\hline & {$[2] \mathrm{PF}_{6}$} & & {$[7] \mathrm{PF}_{6}$} \\
\hline \multicolumn{4}{|l|}{ Interatomic distances } \\
\hline Ru-N1 & $2.099(3)$ & $\mathrm{Rh}-\mathrm{N} 1$ & $2.08(1)$ \\
\hline $\mathrm{Ru}-\mathrm{N} 2$ & $2.089(3)$ & $\mathrm{Rh}-\mathrm{N} 2$ & $2.16(5)$ \\
\hline $\mathrm{Ru}-\mathrm{Cl} 1$ & $2.406(2)$ & $\mathrm{Rh}-\mathrm{Cl} 1$ & $2.396(4)$ \\
\hline Ru-centroid (C6 ring) & 1.683 & Rh-centroid (C5 ring) & 1.79 \\
\hline C5-C6 & $1.351(5)$ & $\mathrm{C} 5-\mathrm{C} 6$ & $1.31(1)$ \\
\hline $\mathrm{N} 1-\mathrm{C} 1$ & $1.390(5)$ & $\mathrm{N} 1-\mathrm{C} 1$ & $1.39(2)$ \\
\hline $\mathrm{N} 2-\mathrm{N} 3$ & $1.420(5)$ & N2-N3 & $1.42(1)$ \\
\hline \multicolumn{4}{|l|}{ Angles } \\
\hline $\mathrm{N} 1-\mathrm{Ru}-\mathrm{N} 2$ & $75.50(1)$ & N1-Rh-N2 & $73.25(3)$ \\
\hline N1-Ru-Cl1 & $83.80(1)$ & N1-Rh-Cl1 & $86.21(3)$ \\
\hline N2-Ru-Cl1 & $84.52(1)$ & $\mathrm{N} 2-\mathrm{Rh}-\mathrm{Cl} 1$ & $91.47(2)$ \\
\hline Ru1-N1-C5 & $116.90(2)$ & Rh1-N1-C5 & $115.21(8)$ \\
\hline Ru1-N2-C6 & $115.50(2)$ & Rh1-N2-C6 & $114.72(4)$ \\
\hline
\end{tabular}

\section{Results and discussion}

\subsection{Synthesis of ligands}

The ligands 3-chloro-6-(3-pyridyl-1-pyrazolyl)pyridazine $(\mathrm{pp}-\mathrm{Cl})$ and 3,6-bis(3-pyridyl-1-pyrazolyl)pyridazine were synthesized by the condensation of 3,6dichloropyridazine and 3-(2-pyridyl)-1H-pyrazole. The reaction was carried out in acetone under refluxing condition in the presence of potassium carbonate and the phase transfer catalyst tetrabutylammonium bromide. The resulting compounds were characterized by spectroscopic methods.

\subsection{Synthesis of the mononuclear complexes $[\mathbf{1}-\mathbf{8}] P F_{6}$}

The mononuclear cationic arene ruthenium and pentamethylcyclopentadienyl rhodium and iridium complexes having 3-chloro-6-(3-pyridyl-1-pyrazolyl)pyridazine $(\mathrm{pp}-\mathrm{Cl})$ and 3,6-bis(3-pyridyl-1-pyrazolyl) pyridazine (bppp) ligands viz., $\left[\left(\eta^{6}-\mathrm{C}_{6} \mathrm{H}_{6}\right) \mathrm{RuCl}(p p-C l)\right]$ $\mathrm{PF}_{6} \quad[\mathbf{1}] \mathrm{PF}_{6},\left[\left(\eta^{6}-p-{ }^{\mathrm{i}} \mathrm{PrC}_{6} \mathrm{H}_{4} \mathrm{Me}\right) \mathrm{RuCl}(p p-C l)\right] \mathrm{PF}_{6}$ [2] $\mathrm{PF}_{6}, \quad\left[\mathrm{Cp}^{*} \mathrm{RhCl}(p p-C l)\right] \mathrm{PF}_{6} \quad[3] \mathrm{PF}_{6}$ and $\left[\mathrm{Cp}^{*} \mathrm{IrCl}\right.$ $(p p-C l)] \mathrm{PF}_{6}[4] \mathrm{PF}_{6}$ (scheme 1) and $\left[\left(\eta^{6}-\mathrm{C}_{6} \mathrm{H}_{6}\right) \mathrm{RuCl}\right.$ $($ bppp $)] \mathrm{PF}_{6} \quad[5] \mathrm{PF}_{6}, \quad\left[\left(\eta^{6}-p-{ }^{\mathrm{i}} \mathrm{PrC}_{6} \mathrm{H}_{4} \mathrm{Me}\right) \mathrm{RuCl}(b p p p)\right]$ $\mathrm{PF}_{6}[6] \mathrm{PF}_{6},[\mathrm{Cp} * \mathrm{RhCl}(b p p p)] \mathrm{PF}_{6}[7] \mathrm{PF}_{6}$ and $\left[\mathrm{Cp}^{*}\right.$

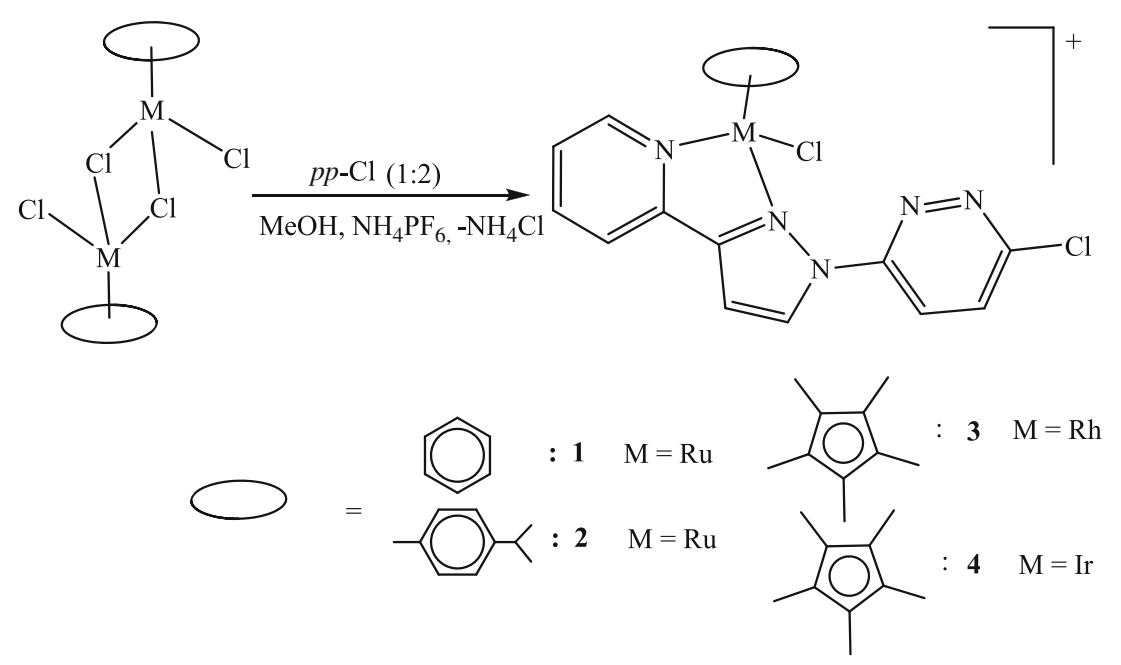

Scheme 1. The mononuclear cationic arene ruthenium and pentamethylcyclopentadienyl rhodium and iridium complexes having ( $\mathrm{pp}-\mathrm{Cl}$ ) ligand. 
$\operatorname{IrCl}($ bppp) $] \mathrm{PF}_{6}[\mathbf{8}] \mathrm{PF}_{6}$ (scheme 2) have been prepared by the reaction of arene or pentamethylcyclopentadienyl complexes $\left[\left(\eta^{6} \text {-arene }\right) \mathrm{Ru}(\mu-\mathrm{Cl}) \mathrm{Cl}\right]_{2}$ (arene $=$ $\mathrm{C}_{6} \mathrm{H}_{6}$ and $p$ - $\left.{ }^{\mathrm{i}} \mathrm{PrC}_{6} \mathrm{H}_{4} \quad \mathrm{Me}\right)$ and $\left[\left(\mathrm{Cp}^{*}\right) \mathrm{M}(\mu-\mathrm{Cl}) \mathrm{Cl}\right]_{2}$ $(\mathrm{M}=\mathrm{Rh}$ and $\mathrm{Ir})$ with 2.1 equivalents of ligand 3chloro-6-(1-pyridyl-3-pyrazolyl)pyridazine $(\mathrm{pp}-\mathrm{Cl})$ or 3,6-bis(1-pyridyl-3-pyrazolyl)-pyridazine (bppp) in methanol. The complexes $\mathbf{1}$ to $\mathbf{8}$ were isolated as hexafluorophosphate salts and exhibit an orange-red colour. They are non-hygroscopic, air-stable, shiny crystalline solids sparingly soluble in methanol, dichloromethane and chloroform, but soluble in acetone and acetonitrile. All these metal complexes were fully characterized by IR, NMR and UV-Vis and mass spectrometry. The infrared spectra of the complexes $\mathbf{1}$ to 8 exhibit a strong band in the region $844-850 \mathrm{~cm}^{-1}$, a typical $v_{(\mathrm{P}-\mathrm{F})}$ stretching band and for the $\mathrm{PF}_{6}$ anions as well as bands corresponding phenyl, pyridyl, pyrazolyl and pyridazine $(\mathrm{C}=\mathrm{C}$ and $\mathrm{C}=\mathrm{N})$ rings were observed. The mass spectra of these compounds exhibited the corresponding molecular ion peaks at $\mathrm{m} / \mathrm{z}=$ 472, 527, 531, 621, 581, 637, 639 and 729. The ${ }^{1} \mathrm{H}$ NMR spectrum of free ligands 3-chloro-6-(3-pyridyl1-pyrazolyl)pyridazine ( $\mathrm{pp}-\mathrm{Cl}$ ) exhibits a characteristic set of eight resonances at $8.77(\mathrm{~d}, 1 \mathrm{H}), 8.68(\mathrm{~d}, 1 \mathrm{H})$, $8.34(\mathrm{~d}, 1 \mathrm{H}), 8.04(\mathrm{~d}, 2 \mathrm{H}), 7.77(\mathrm{dt}, 1 \mathrm{H}), 7.63(\mathrm{~d}, 2 \mathrm{H})$, $7.28(\mathrm{dt}, 1 \mathrm{H}), 7.18(\mathrm{~d}, 1 \mathrm{H})$ and 3,6-bis(3-pyridyl-1pyrazolyl)pyridazine ( at $\delta=8.81(\mathrm{~d}, 2 \mathrm{H}), 8.71(\mathrm{~d}, 2 \mathrm{H}), 8.52(\mathrm{~s}, 2 \mathrm{H}), 8.11$ $(\mathrm{d}, 2 \mathrm{H}), 7.80(\mathrm{dt}, 2 \mathrm{H}), 7.29(\mathrm{dt}, 2 \mathrm{H}), 7.21(\mathrm{~d}, 2 \mathrm{H})$ for the pyrazole, pyridazine and pyridine ring protons. Upon coordination with the metal atom, the cationic complexes $\mathbf{1}$ to $\mathbf{4}$ exhibit five to six distinct resonances at $8.91(\mathrm{~d}, 1 \mathrm{H}), 8.67(\mathrm{~d}, 1 \mathrm{H}), 8.50(\mathrm{~d}, 1 \mathrm{H}), 8.36(\mathrm{~m}$, $3 \mathrm{H}), 7.44(\mathrm{dt}, 1 \mathrm{H}), 7.22(\mathrm{~d}, 1 \mathrm{H})$ which are assignable to pyrazolyl, pyridazine and pyridyl ring protons of the 3chloro-6-(3-pyridyl-1-pyrazolyl)pyridazine ( $\mathrm{pp}-\mathrm{Cl}$ ) ligand and complexes $\mathbf{5}$ to $\mathbf{8}$ exhibit in between seven and nine distinct resonances at $9.55(\mathrm{~d}, 1 \mathrm{H}), 8.72(\mathrm{~d}, 1 \mathrm{H})$, $8.68(\mathrm{~d}, 1 \mathrm{H}), 8.20-8.18(\mathrm{~m}, 4 \mathrm{H}), 7.68(\mathrm{dt}, 2 \mathrm{H}), 7.42$ (t, 1H), $7.38(\mathrm{~d}, 1 \mathrm{H}), 7.22(\mathrm{~d}, 1 \mathrm{H}), 7.18(\mathrm{~d}, 2 \mathrm{H})$ which are assignable to pyrazolyl, pyridazine and pyridyl ring protons of the 3,6-bis(3-pyridyl-1-pyrazolyl)pyridazine (bppp) ligand, indicating formation of mononuclear complexes. Besides these resonances, complexes 1 and 5 exhibit a singlet at $\delta=5.95$ and 5.92, respectively for the protons of the benzene ligand. Complexes 2 and 6 exhibits two doublets at $\delta=1.71-1.69$, as well as a septets at $\delta=2.70-2.32$ for the protons of the isopropyl group and a singlet at $2.17 \mathrm{ppm}$ for the methyl protons of $p$-cymene ring. The four doublets observed at 5.59-5.39 correspond to the aromatic $p$-cymene ring $\mathrm{CH}$ protons. This unusual pattern is due to the diastereotopic methyl protons of the isopropyl group and aromatic protons of the $p$-cymene ligand, since the ruthenium atom is stereogenic due to the coordination of four different ligand atoms and chiral nature of metal atom. ${ }^{27-29}$ Interestingly, the chemical shifts of complex $\mathbf{6}$ shows downfield compared to complex $\mathbf{2}$ of $p$-cymene ligand. Complexes 3, 4, 7 and 8 exhibit a

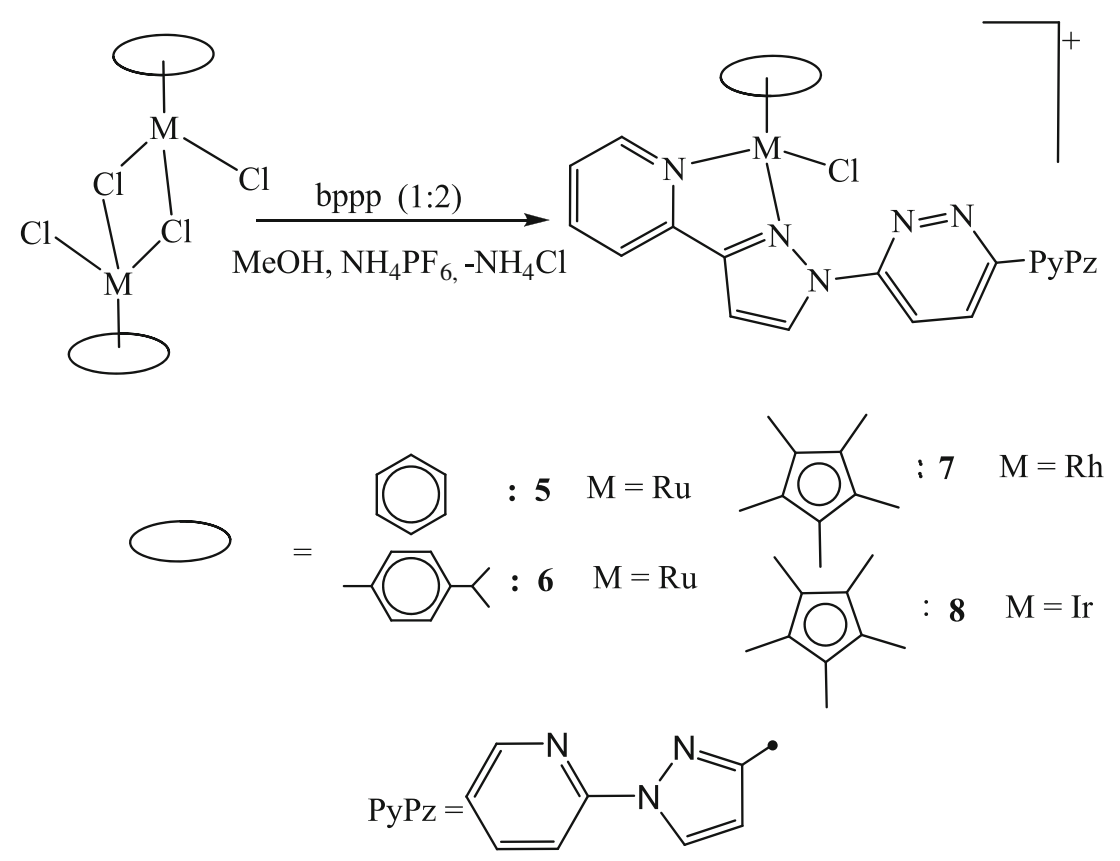

Scheme 2. The mononuclear cationic arene ruthenium and pentamethylcyclopentadienyl rhodium and iridium complexes having (bppp) ligand. 
strong peak at $\delta=2.15,1.88,2.11$ and 1.98 for pentamethylcyclopentadienyl ligand, respectively, which are slightly shifted downfield in comparison to the starting complexes.

\subsection{Crystal structure analysis of $\left[\left(\eta^{6}-p-{ }^{\mathrm{i}} \mathrm{PrC}_{6} \mathrm{H}_{4} \mathrm{Me}\right) \mathrm{Ru}(\mathrm{pp}-\mathrm{Cl}) \mathrm{Cl}\right] \mathrm{PF} \mathrm{F}_{6}\left([\mathbf{2}] \mathrm{PF}_{6}\right)$ and $[\mathrm{Cp} * R h(b p p p) C l] P F_{6}\left([7] P F_{6}\right)$}

The molecular structure of $\left[\left(\eta^{6}-p-{ }^{\mathrm{i}} \mathrm{PrC}_{6} \mathrm{H}_{4} \mathrm{Me}\right) \mathrm{Ru}-\right.$ $(p p-C l) \mathrm{Cl}] \mathrm{PF}_{6} \quad\left([2] \mathrm{PF}_{6}\right)$ and $\quad\left[\mathrm{Cp}^{*} \mathrm{Rh}(b p p p) \mathrm{Cl}\right] \mathrm{PF}_{6}$ [7] $\mathrm{PF}_{6}$ have been established by single-crystal $\mathrm{X}$-ray structure analysis. Both complexes shown a typical piano-stool geometry with the metal centre coordinated by the aromatic ligand, chloride and a chelating $N, N^{\prime}$-ligand (see figures 1 and 2). The metal atom is in octahedral arrangement and the $\mathrm{pp}-\mathrm{Cl}$ or bppp ligand is found to coordinate through the $\mathrm{N} 1$ atom of the pyridine moiety and the $\mathrm{N} 2$ atom of the pyrazolyl ring to generate a five-membered ring metallo-cycle (see figures 1 and 2). In these complexes, the $\mathrm{N}$ atom of pyridazine points away from the metal centre and show no interaction with neighbouring cations. Selected bond lengths and angles for complexes $\mathbf{2}$ and $\mathbf{7}$ are presented in table 2. In the mononuclear complex $\mathbf{2}$ the N1-metal [2.099(3) $\AA$ ] distance of the pyridyl ring is slightly longer than the corresponding pyrazolyl, N2-metal distance [2.089(3) $\AA$ ], in contrast, for complex 7 the N1-metal [2.082(1) $\AA$ ] distance slightly shorter than the corresponding pyrazolyl N2-metal distance [2.161(5) $\AA$ ], which are comparable to those in $\left[\left(\eta^{6}-\mathrm{C}_{6} \mathrm{Me}_{6}\right) \mathrm{RuCl}\left(\mathrm{C}_{5} \mathrm{H}_{4} \mathrm{~N}-2-\mathrm{CH}=\mathrm{N}=\mathrm{C}_{6} \mathrm{H}_{4}-p-\mathrm{NO}_{2}\right)\right]$ $\mathrm{PF}_{6}{ }^{30}, \quad\left[\mathrm{Ru}(\mathrm{mes}) \mathrm{Cl}\left\{\mathrm{C}_{5} \mathrm{H}_{4} \mathrm{~N}-2-\mathrm{C}(\mathrm{Me})=\mathrm{N}(\mathrm{CHMePh})\right\}\right]$

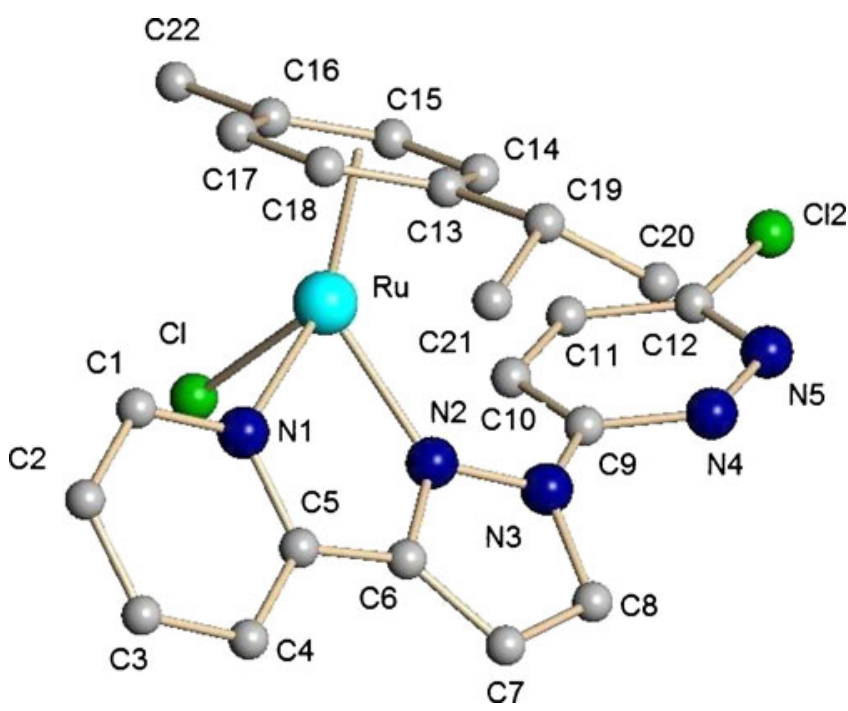

Figure 1. Molecular structure of complex 2. Hydrogen atoms and anion are omitted for clarity.
$\mathrm{BF}_{4}{ }^{31}, \quad\left[\mathrm{Cp} * \mathrm{RhCl}\left(\mathrm{C}_{5} \mathrm{H}_{4} \mathrm{~N}-2-\mathrm{CH}=\mathrm{N}=\mathrm{C}_{6} \mathrm{H}_{4}-p-\mathrm{NO}_{2}\right)\right]$ $\mathrm{BF}_{4}{ }^{32}, \quad\left[\left(\eta^{6}-\mathrm{C}_{6} \mathrm{H}_{6}\right) \mathrm{Ru}(2\right.$-(2-thiazolyl)-1,8-naphthyridine $) \mathrm{Cl}] \mathrm{PF}_{6},{ }^{27} \quad\left[\left(\eta^{6}-p-{ }^{-} \mathrm{PrC}_{6} \mathrm{H}_{4} \mathrm{Me}\right) \quad \mathrm{RuCl}(2,3-\right.$ bis (2-pyridyl)pyrazine) $\mathrm{BF}_{4} \cdot{ }^{33}$ While the $\mathrm{M}-\mathrm{Cl}$ [2.406(2) $\AA$ and 2.396(4) $\AA$ ] bond lengths show no significant differences in all the cations and reported values. ${ }^{34-37}$ The $\mathrm{N}(1)-\mathrm{M}(1)-\mathrm{N}(2)$ bond angle in complex 2 and 7 is found to be [75.1(1) $\left.{ }^{\circ}\right]$ and $\left[73.2(3)^{\circ}\right]$, respectively, which are similar to those of complexes $\quad\left[\left(\eta^{6}-p-{ }^{i} \mathrm{PrC}_{6} \mathrm{H}_{4} \mathrm{Me}\right) \mathrm{RuCl}(2,3\right.$-bis(2-pyridyl)

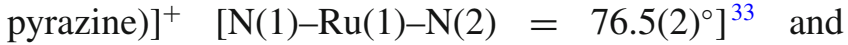
$\left[\left(\eta^{6}-p-{ }^{\mathrm{i}} \mathrm{PrC}_{6} \mathrm{H}_{4} \mathrm{Me}\right) \mathrm{RuCl}(2,3-\right.$ bis $(2-p y r i d y l) q u i n o-$ xaline) $]^{+}{ }^{37}$ The distances between the ruthenium atom and the centroid of the $\left(\eta^{6}-p-{ }^{i} \operatorname{PrC}_{6} \mathrm{H}_{4} \mathrm{Me}\right)$ ring is $1.683 \AA$ in complex 2 , whereas the distance between the rhodium atom and the centroid of the $\eta^{5}-\mathrm{C}_{5} \mathrm{Me}_{5}$ ring is $1.781 \AA$ in complex 7. These bond distances are comparable to those in the related complex cations $\left[\left(\eta^{6}-p-{ }^{i} \mathrm{PrC}_{6} \mathrm{H}_{4} \mathrm{Me}\right) \mathrm{Ru}(\mathrm{pyNp}) \mathrm{Cl}\right] \mathrm{PF}_{6}$, $[\mathrm{Cp} * \operatorname{Ir}(\mathrm{pyNp}) \mathrm{Cl}] \mathrm{PF}_{6} \quad(\mathrm{PyNp}=2$-(2-pyridyl)-1,8-naphthyridine) $(1.79 \AA)^{27}$ and [Cp* $\mathrm{Rh}(3,6$-bis(2-pyridyl)-4phenylpyridazine) $\mathrm{Cl} \mathrm{PF}_{6}(1.789 \AA)$.

\subsection{Synthesis of the dinuclear complexes [9-12] $\left(P F_{6}\right)_{2}$}

The reaction of the dimeric chloro complexes $\left[\left(\eta^{6}-\right.\right.$ arene $) \mathrm{Ru}(\mu-\mathrm{Cl}) \mathrm{Cl}]_{2}\left(\right.$ arene $\left.=\mathrm{C}_{6} \mathrm{H}_{6}, p-{ }^{\mathrm{i}} \mathrm{PrC}_{6} \mathrm{H}_{4} \mathrm{Me}\right)$ and $[\mathrm{Cp} * \mathrm{M}(\mu-\mathrm{Cl}) \mathrm{Cl}]_{2}(\mathrm{M}=\mathrm{Rh}, \mathrm{Ir})$ with 1 equivalent of 3,6-bis(3-pyridyl-1-pyrazolyl)pyridazine ( methanol results in the formation of the orange colour, air-stable dinuclear complexes $\left[\left\{\left(\eta^{6}-\mathrm{C}_{6} \mathrm{Me}_{6}\right) \mathrm{RuCl}\right\}_{2}\right.$ $($ bppp $)]\left(\mathrm{PF}_{6}\right)_{2} \quad[9]\left(\mathrm{PF}_{6}\right)_{2}, \quad\left[\left\{\left(\eta^{6}-p-{ }^{\mathrm{i}} \mathrm{PrC}_{6} \mathrm{H}_{4} \mathrm{Me}\right) \mathrm{RuCl}\right\}_{2}\right.$ $($ bppp $)]\left(\mathrm{PF}_{6}\right)_{2}[\mathbf{1 0}]\left(\mathrm{PF}_{6}\right)_{2},\left[\left\{\mathrm{Cp}^{*} \mathrm{RhCl}\right\}_{2}((b p p p)]\left(\mathrm{PF}_{6}\right)_{2}\right.$ $[11]\left(\mathrm{PF}_{6}\right)_{2}$ and $\left[\{\mathrm{Cp} * \mathrm{IrCl}\}_{2}((b p p p)]\left(\mathrm{PF}_{6}\right)_{2}[\mathbf{1 2}]\left(\mathrm{PF}_{6}\right)_{2}\right.$. All these complexes are isolated as their hexafluorophosphate salts (scheme 3) and they were characterized by IR, Mass, ${ }^{1} \mathrm{H}-\mathrm{NMR}$ spectrometry and elemental analysis. Infrared spectra of these dinuclear complexes 9 to $\mathbf{1 2}$, showed a similar trend to the mononuclear cationic complexes $\mathbf{1}$ to $\mathbf{8}$. In the mass spectra the complexes 7, 8, 11 and 12 hexafluorophosphate salts give rise to two main peaks; a minor peak with an approximately $50 \%$ intensity attributed to $\left[\mathrm{M}^{2+}+\mathrm{PF}_{6}^{-}\right]^{+}$ at $\mathrm{m} / \mathrm{z}$ 940, 1052, 1058 and 1236, respectively and a major peak which corresponds to loss of $[(\text { arene }) \mathrm{MCl}]^{+}$ fragment to the formation of mononuclear cations 5-8 at $\mathrm{m} / \mathrm{z}=580,637,739$ and 729 , respectively. The ${ }^{1} \mathrm{H}$ NMR spectra of the dinuclear cationic complexes 9 to 12 exhibit seven distinct resonances at 9.55 (d, 2H), $9.37(\mathrm{~s}, 2 \mathrm{H}), 9.14(\mathrm{~d}, 2 \mathrm{H}), 8.52(\mathrm{~d}, 2 \mathrm{H}), 8.48$ $(\mathrm{t}, 2 \mathrm{H}), 8.36(\mathrm{t}, 2 \mathrm{H}), 7.86(\mathrm{~d}, 2 \mathrm{H})$ which are assignable 


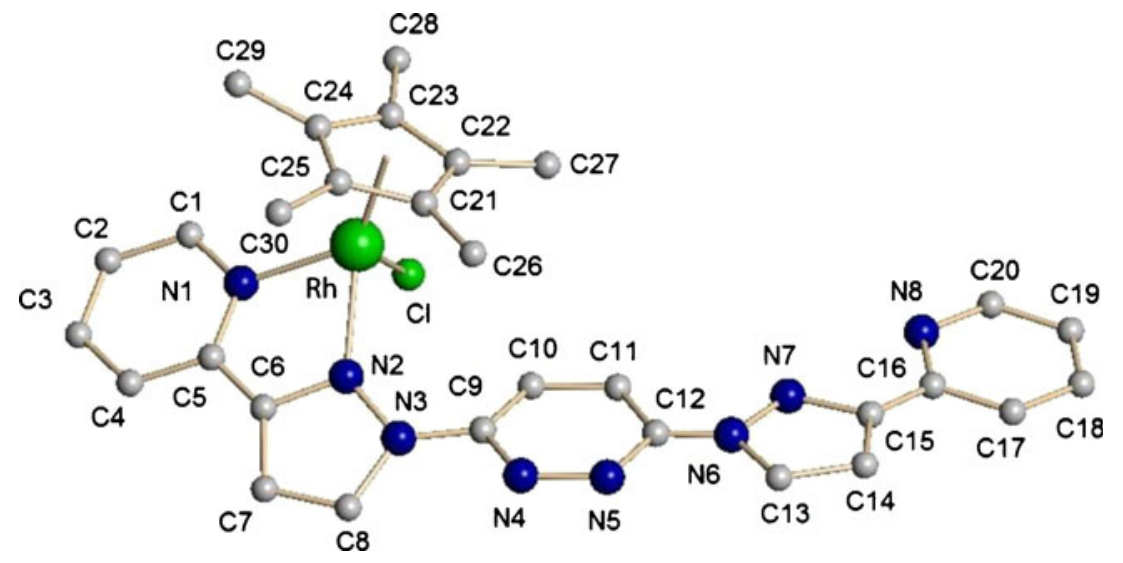

Figure 2. Molecular structure of complex 7. All hydrogen atoms, solvent molecule and anion are omitted for clarity.

to pyridine, pyrazole and pyridazine ring protons of the 3,6-bis(3-pyridyl-1-pyrazolyl)pyridazine ( The chemical shift of the bppp ligand protons upon complex formation shifted down field with reference to free ligand. However, $\mathrm{H}_{\mathrm{ff}}$ protons of metal bounded pyrazoles shifted up field compared to $\mathrm{H}_{\mathrm{gg}}$ unbound pyridazine protons of bppp ligand up on complexation; this may be due to the metal to ligand charge transfer in all these complexes (figure 3). Besides these bppp ligand resonances complex 9 exhibits a singlet at $\delta=$ 5.75 for the two benzene rings and complex 10 exhibits two doublets at $\delta=1.18-1.12$, and septet at $\delta=2.68$ for the protons of the isopropyl group and a singlet at $\delta=2.25$ for the methyl protons of $p$-cymene ring. The four doublets observed at $\delta=6.10-5.54$ correspond to the aromatic $p$-cymene ring $\mathrm{CH}$ protons. Interestingly, the chemical shift of these protons as well as methyl protons shifted downfield significantly with reference to starting precursor ranging from $\delta=5.20$ to 5.42 and mono nuclear complexes (figure 3 ). This could be due to the increased steric nature on the $p$-cymene ring in dinuclear complexes compared to mono nuclear compounds. Complexes $\mathbf{1 1}$ and $\mathbf{1 2}$ exhibit a strong peak at $\delta=2.05$ and 1.99 for the protons of pentamethylcyclopentadienyl ligands, respectively which are slightly shifted downfield in comparison to the starting complexes. Due to lack of formation of single crystals of these complexes, we are assigned by NMR spectral data, these are dinuclear complexes bridged by the ligand bppp and formation of dicationic complexes.

\subsection{UV-visible spectroscopy}

Electronic absorption spectra of compounds $\mathrm{pp}-\mathrm{Cl}$, bppp and complexes $\mathbf{1}$ to $\mathbf{1 2}$ were acquired in acetonitrile, at $10^{-5} \mathrm{M}$ concentration in the range of

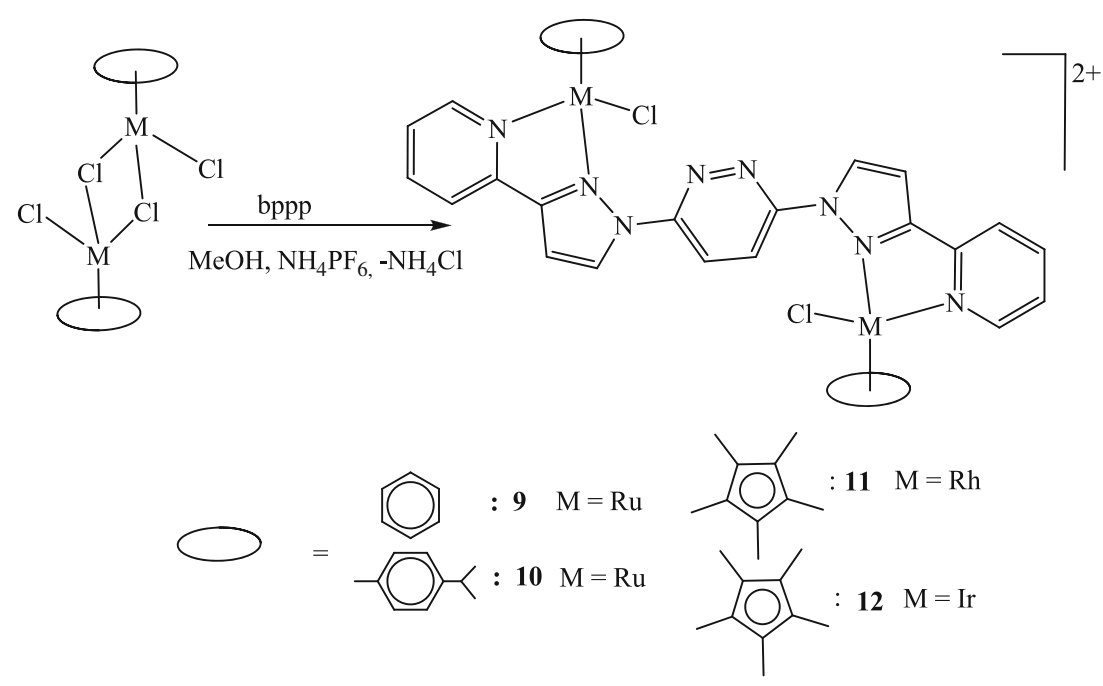

Scheme 3. The dinuclear cationic arene ruthenium and pentamethylcyclopentadienyl rhodium, iridium complexes having (bppp) ligand. 


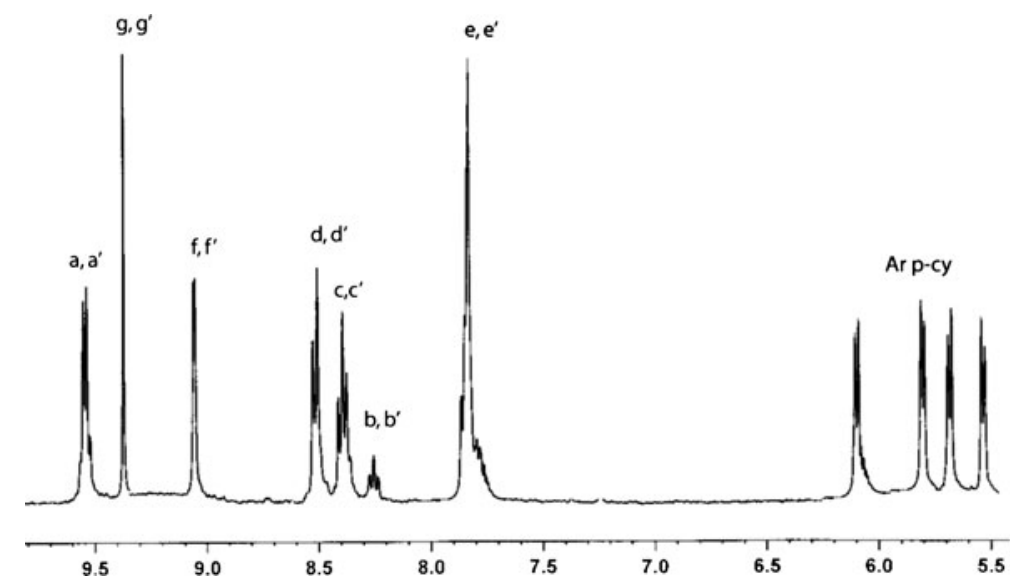

Figure 3. ${ }^{1} \mathrm{H}-\mathrm{NMR}$ spectrum of complex $\mathbf{1 0}$ in $\mathrm{CDCl} 3$, represents bppp ligand resonances and $p$-cymene ligand aromatic protons (isopropyl protons are omitted for clarity).

220-550 nm. The spectral data are well-formulated in experimental section. The spectrum of the ligand 3-chloro-6-(3-pyridyl-1-pyrazolyl)pyridazine ( $p p$ $\mathrm{Cl}$ ) exhibits a band at $318 \mathrm{~nm}$, while ligand 3,6-bis(3pyridyl-1-pyrazolyl)pyridazine ( $b p p p$ ) exhibits two bands at $316 \mathrm{~nm}$ and $331 \mathrm{~nm}$ as a shoulder peak, which are assigned to intra-ligand $\pi \rightarrow \pi^{*}$ transitions. The electronic spectra of these complexes are characterized by two main features, viz., an intense ligand-localized or intra ligand $\pi \rightarrow \pi^{*}$ transition in the ultraviolet region and metal-to-ligand charge transfer (MLCT) $\mathrm{d} \pi(\mathrm{M}) \rightarrow \pi^{*}$ (L - ligand) bands in the visible region. ${ }^{38}$ Since the low spin $\mathrm{d}^{6}$ configuration of the mononuclear complexes provides filled orbitals of proper symmetry at the $\mathrm{Ru}(\mathrm{II}), \mathrm{Rh}(\mathrm{III})$ and $\mathrm{Ir}(\mathrm{III})$ centres, these can interact with low-lying $\pi^{*}$ orbitals of the ligands. All the mononuclear complexes $\mathbf{1}$ to $\mathbf{4}$ show only an intense band in the region of 308-316 nm, while complexes 5

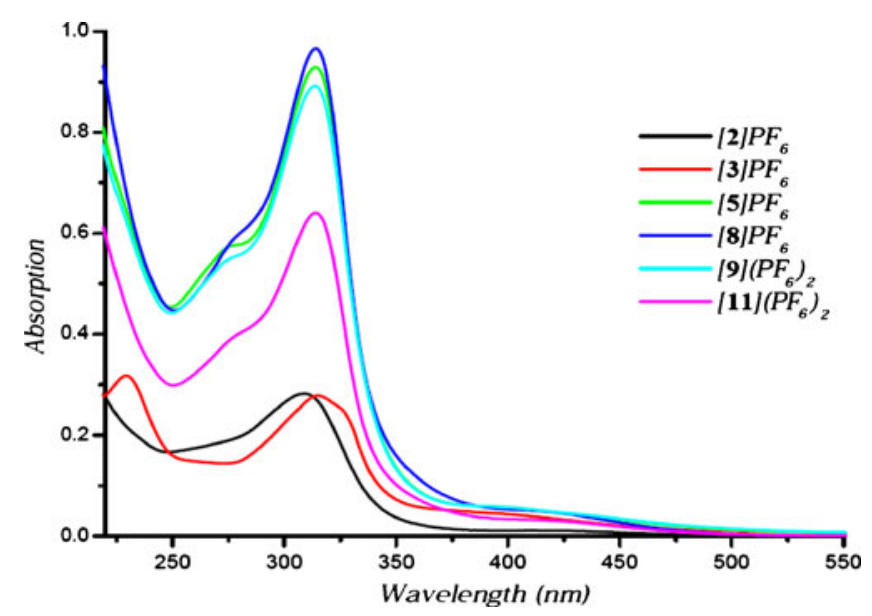

Figure 4. UV-visible electronic spectra of representative complexes in acetonitrile at $298 \mathrm{~K}$. to $\mathbf{8}$ shown three bands at $276-278 \mathrm{~nm}, 312-314 \mathrm{~nm}$ and a broad peak at $417-423 \mathrm{~nm}$. Whereas the dinuclear complexes 9 to 12 show three bands, which are almost similar to complexes $\mathbf{5}$ to $\mathbf{8}$ at 274 to $276 \mathrm{~nm}$ as a shoulder peak at $312-314 \mathrm{~nm}$ as a high intense peak and a broad band at $418-420 \mathrm{~nm}$. The high intensity band in UV region for both mononuclear and dinuclear complexes is assigned to inter and intra-ligand $\pi-\pi * / n-\pi *$ transitions, ${ }^{39-41}$ while the low energy absorption band in the visible region for all complexes is assigned to metal-to-ligand charge transfer (MLCT) $\left(t_{2 g}-\pi^{*}\right)$. Representative spectra of these complexes are represented in figure 4 .

\section{Conclusions}

In summary, we have prepared two novel chelating ligands 3-chloro-6-(3-pyridyl-1-pyrazolyl)pyridazine $(\mathrm{pp}-\mathrm{Cl})$ and 3,6-bis(3-pyridyl-1-pyrazolyl)pyridazine (bppp). Ligands $p p-C l$ and bppp reacted with series of arene ruthenium and $\mathrm{Cp}^{*}$ rhodium and iridium complexes giving new series of mononuclear and binuclear complexes. However, we were unable to get single crystals of dinuclear complexes, which were characterized by other spectral techniques.

\section{Supplementary data}

CCDC-749700 [2] $\mathrm{PF}_{6}$ and CCDC-749701 [7]PF 6 contain the crystallographic data for this paper. These data can be obtained free of charge via www.ccdc. cam.ac.uk/data_request/cif or by e-mailing data request@ccdc.cam.ac.uk, or by contacting: The Cambridge Crystallographic Data Centre, 12 Union 
Road, Cambridge CB2 1EZ, UK; fax: +44 1223 336033.

\section{Acknowledgements}

The authors thank the University Grants Commission (UGC), New Delhi for providing the financial support in the form of financial grant No. 39-793/2010 (SR) and for research fellowship (UGC-RFSMS).

\section{References}

1. Trost B M, Toste F D and Pinkerton A B 2001 Chem. Rev. 1012067

2. Touge T, Hakamata T, Nara H, Kobayashi T, Sayo N, Saito T, Kayaki Y and Ikariya T $2011 \mathrm{~J}$. Am. Chem. Soc. 13314960

3. Ito M, Ootsuka T, Watari R, Shiibashi A, Himizu A and Ikariya T 2011 J. Am. Chem. Soc. 1334240

4. Noyori R and Hashigushi S 1997 Acc. Chem. Res. 30 97, and references therein

5. Jauregui-Haja U J, Dessoudeix M, Kalck P, Wilhelm M and Delmas H 2001 Catl. Today. 66297

6. Styring P, Grindon C and Fisher C M 2001 Catal. Lett. 77219

7. Kimura T, Koiso N, Ishiwata K, Kuwata S and Ikariya T 2011 J. Am. Chem. Soc. 1338880

8. Ang W H and Dyson P J 2006 Eur. J. Inorg. Chem. 4003

9. Auzias M, Therrien B, Süss-Fink G, Štěpnička P, Ang W H and Dyson P J 2008 Inorg. Chem. 47578

10. Habtemariam A, Melchart M, Fernandez R, Parsons S, Oswald I D H, Parkin A, Fabbiani F P A, Davidson J E, Dawson A, Aird R E, Jodrell D I and Sadler P J 2006 J. Med. Chem. 496858

11. Melchart M, Habtemariam A, Novakova O, Moggach S A, Fabbiani F P A, Parsons S, Brabec V and Sadler P J 2007 Inorg. Chem. 468950

12. Scolaro C, Bergamo A, Brescacin L, Delfino R, Cocchietto M, Laurenczy G, Geldbach T J, Sava G and Dyson P J 2005 J. Med. Chem. 484161

13. Hilllard E, Vessieres A, Le Bideau F, Plazuk d, Spera d, Huche M and Jaouen G 2006 Chem. Med. Chem. 1551

14. Auzias M, Therrien B, Suss-Fink G, Šěpnička P, Ang W H and Dyson P J 2008 Inorg. Chem. 47578

15. Haga M, Ali M M, Maegawa H, Nozaki K, Yoshimura A and Ohno T 1994 Coord. Chem. Rev. 9499

16. Haga M, Ali M M and Arakava R 1996 Angew. Chem. Int. Ed. Engl. 3576

17. Baitalik S, Florke U and Nag K 1999 Inorg. Chem. 38 3296
18. Williams R L, Toft H N, Winkel B and Brewer K J 2003 Inorg. Chem. 424394

19. Scott S M, Gordon K C and Burrell A K 1999 J. Chem. Soc., Dalton Trans. 2669

20. Marcaccio M, Paolucci F, Paradisi C, Roffia S, Fontanesi C, Yellowlees L J, Serroni S, Campagna S, Denti G and Balzani V 1999 J. Am. Chem. Soc. 12110081

21. Kirchhoff J R and Kirschbaum K 1998 Polyhedron 17 4033

22. Bennett M A, Huang T N, Matheson T W and Smith A K 1982 Inorg. Synth. 2174

23. White C, Yates A and Maitlis P M 1992 Inorg. Synth. 29 228

24. Amoroso A J, Thompson A M C, Jeffery J C, Jones P L, McCleverty J A and Ward M D 1994 J. Chem. Soc., Chem. Commun. 2751

25. XRD 2002 Single-crystal Software; Bruker Analytical $x$-ray Systems, (Madison, WI: USA)

26. Sheldrick G M 1999 SHELXS-97 and SHELXL- 97, University of Göttingen, Göttingen, Germany

27. Prasad K T, Therrien B and Rao K M 2008 J. Organomet. Chem. $\mathbf{6 9 3} 3049$

28. Davies D L, Fawcett J, Krafczyk R, Russell D R and Singh K 1998 Dalton Trans. 2349

29. Davies D L, -Duaij O A, Fawcett J, Giardiello M, Hilton S T and Russell D R 2003 Dalton Trans. 4132

30. Lalrempuia R, Carroll P J and Rao K M 2003 Polyhedron 22605

31. Davies D L, Fawcett J, Krafczyk R and Russell D R 1997 J. Organomet. Chem. 545-546 581

32. Govindaswamy P, Mozharivskyj Y A and Rao K M 2005 Polyhedron 241710

33. Singh A, Singh N and Pandey D S 2002 J. Orgnomet. Chem. 64248

34. Therrien B, Said-Mohamed C and Suss-Fink G 2008 Inorg. Chim. Acta. 3612601

35. Prasad K T, Gupta G, Rao A V, Das B and Rao K M 2009 Polyhedron 282649

36. Gupta G, Prasad K T, Das B, Yap G L P and Rao K M 2009 J. Organomet. Chem. 6942618

37. Lalrempuia R and Rao K M 2003 Polyhedron 22 3155

38. B-Soriaga E, Keder N L and Kaska W C 1990 Inorg. Chem. 293167

39. Govindaswamy P, Canivet J, Therrien B, Süss-Fink G, Štěpnička P and Ludvík J 2007 J. Organomet. Chem. 6923664

40. Arau' jo C S, Drew M G B, Félix V, Jack L, Madureira J, Newell M, Roche S, Santos T M, Thomas J A and Yellowlees L 2002 Inorg. Chem. 412250

41. Deng H, Li J, Zheng K C, Yang Y, Chao H and Ji L N 2005 Inorg. Chim. Acta 3583430 